\title{
Dispersion Characteristics of Electromagnetic Wave in Magnetized One Dimensional Ferrite Photonic Crystals with Mixed Field Configuration
}

YOGESH SHARMA ( $\square$ yogesh_fosc@sgtuniversity.org)

SGT University: Shree Guru Gobind Singh Tricentenary University https://orcid.org/0000-0003-39022226

\section{Surendra Prasad}

Banaras Hindu University Faculty of Science

\section{Research Article}

Keywords: Ferrite photonic crystal Photonic band gaps. Filling factor Dispersion curve. Phase index, Normalized parallel wave vector, External magnetic field

Posted Date: April 27th, 2021

DOI: https://doi.org/10.21203/rs.3.rs-391593/v1

License: (9) (i) This work is licensed under a Creative Commons Attribution 4.0 International License. Read Full License 


\title{
Dispersion Characteristics of Electromagnetic Wave in Magnetized One Dimensional Ferrite Photonic Crystals with Mixed Field Configuration
}

\author{
Yogesh Sharma*, Surendra Prasad \\ *Department of Physics, Faculty of Science, Shree Guru Gobind Singh Tricentenary (SGT) University, Gurgaon- \\ Badli Road Chandu, Budhera, Gurugram, Haryana 122505 INDIA \\ Department of Physics, Institute of Sciences, Banaras Hindu University, Varanasi-221005, India
}

\begin{abstract}
:
The dispersion characteristics and phase index of electromagnetic waves in two types of magnetized one dimensional ferrite photonic crystals with mixed field configuration are studied for transverse magnetic mode. The dispersion equation and formula for phase index are derived using transfer matrix method. It is found that in type-1 $\&$ type-2 magnetized one dimensional ferrite photonic crystals, photonic band gaps (PBGs) show dependence on $H_{0 Y I G}$ and $H_{0 N I}$ respectively. The PBGs corresponding to thinner YIG layer are at higher frequency side at fixed $\beta$. The variation of frequency-filling factor shows that PBGs occur in the form of lobe for both types. It is observed that the phase index is strongly influenced by external magnetic fields, $\beta$ and filling factor. It is demonstrated that PBGs in phase index occur in the form of lobes at fixed $\beta$. Also, with decrease in the magnitude of phase index, number of lobe increases. The phase index, field and frequency plots show that there are multiple peaks in the PBGs of phase index in the lower frequency.
\end{abstract}

Keywords: Ferrite photonic crystal Photonic band gaps. Filling factor Dispersion curve. Phase index, Normalized parallel wave vector, External magnetic field

\section{Introduction}

Over the decades, magneto-optical photonic crystals (MOPhCs) have been active field of extensive research around the globe due to their unique magneto-optical properties. These $\mathrm{MOPhCs}$ structures have shown promising technical applications viz. cavity enhanced Faraday rotation [1-2], non-reciprocal super-prisms [3], ultra compact isolator [4-5], highly-directive miniature antenna arrays [6], non-reciprocal propagation [7], electromagnetic (EM) unidirectionality [8-9], integrated devices [10-11], etc. The EM responses of these composite periodic structures are externally controllable and the dispersion properties of EM waves show external field dependence. These MOPhCs has periodic arrangement of magnetic layers and other media such as dielectrics, semiconductor and metal layers of various geometries. The ferrite based photonic crystals [12-17], also known as ferrite photonic crystals (FPhCs), waveguides [18] and transmission lines [19] are new hot spot in the photonic research due to their unique characteristics. These structures have ferrites as one of the constituent layer in their unit cell. Z. Yu et al. [12] have shown one-way total reflections in one dimensional (1-D) MOPhCs. Ferrite based polarization independent circulator is recently reported [13].

The properties of garnet based MOPhCs are investigated experimentally by A. M. Grishin et al. [14] and have tailored large Faraday rotation and acceptable transmittance from visible to infrared. J.-X. Liu et al. [15] studied the filtering properties of FPhCs. 
*Author to whom correspondence should be addressed. Electronic mail: uvsbhu@ gmail.com

$\mathrm{K}$. Bi et al. [16] have simulated the tunability of dielectric properties of metamaterials based on ferrite-dielectric and verified experimentally. Magnetically tunable circulator in terahertz frequency regime was reported by F. Fan et al. [17]. V. I. Fesenko et al. [18] made extensive study on control of single mode operation in a circular wave guide filled with ferrites. N. Dib et al. [19] simulated the propagation characteristics of cylindrical transmission lines that contain magnetized ferrite material using the finite-difference time-domain method and the spectraldomain analysis.

Very recently, Sharma and Prasad [20-21] have investigated the dispersion properties of EM waves in magnetized 1-D FPhCs structures for transverse electric (TE) and transverse magnetic (TM) modes for various field configurations such as longitudinal [20] and transverse magnetization configurations [21]. They have also explored the EM wave propagation in magnetized 1-D FPhCs structures for TE mode in transverse magnetization configuration [22]. They have shown that phase index and dispersion properties of EM waves can be controlled by external magnetic fields, incident angle and filling factor. Also, these magnetized 1-D FPhCs structures behave as dielectric. In these composite structures, ferrites in the sub-layer of unit cell of 1-D FPhCs structures are either in transverse or longitudinal magnetization configurations. It would be interesting to study dispersion property of EM waves in such structures for mixed field configurations.

In this paper, we considered two types of magnetized 1-D FPhCs structures. In the first type of the structure, we have yttrium iron garnet (YIG) in longitudinal magnetization and nickel (NI) ferrite in transverse magnetization in the unit cell of the 1-D FPhCs structure. In other type, we have swapped the arrangement of the YIG layer and NI ferrites. We have tried to investigate that if we have composite periodic structure with YIG and NI in the unit cell with mixed configurations, then how the dispersion behavior of EM waves will modify. For the analysis, we have used well known transfer matrix method. This paper is organized as follows: in section 1 introduction, in Section 2, theoretical formulation and approach are given. The other necessary formulae used in this paper are also presented. Section 3 is devoted to results and discussion. A conclusion is drawn in section 4 .

\section{Theoretical formulation and approach}

The dispersion behavior of EM waves is investigated in two types of magnetized 1-D FPhCs. The unit cell of type-1 (Fig. 1) 1-D FPhCs has two sub-layers. The sub-layer 1 consists of YIG ferrite which is kept in longitudinal magnetization configuration and sub-layer 2 has NI ferrite which is in transverse magnetization. The type-2 1-D FPhCs (Fig. 2) has NI ferrite in longitudinal magnetization in one sub-layer and YIG ferrite in transverse magnetization in other sub-layer of the unit cell. The thicknesses of YIG and NI ferrites sub-layers in type-1 1-D FPhCs are ' $a$ ' and ' $b$ ' respectively. Type-2 1-D FPhCs has NI and YIG ferrites with thicknesses as ${ }^{\prime} a^{\prime}$ and ${ }^{\prime} b$ ' respectively. 


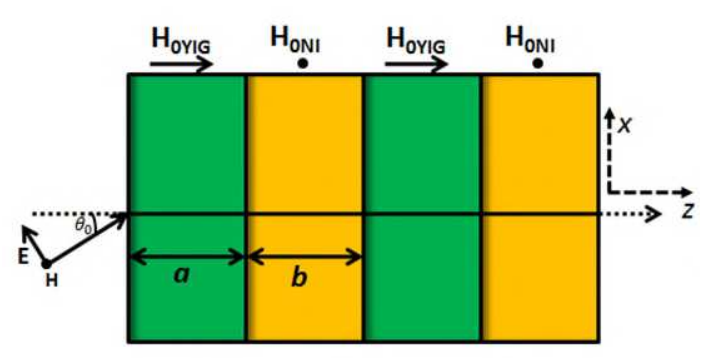

Fig. 1 Schematic diagram of type-1 1-D FPhCs

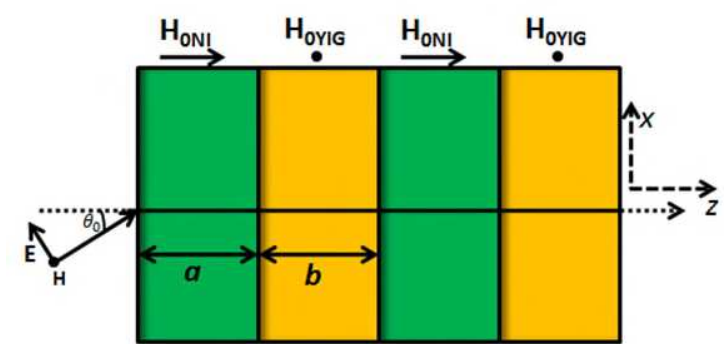

Fig. 2 Schematic diagram of type-2 1-D FPhCs

An EM wave is assumed to be incident obliquely on the surface of the outermost layer making an angle $\theta_{0}$ with respect to normal to the YIG layer. In the case of transverse magnetic (TM) mode, the wave propagation takes place in $x-z$ plane and wave magnetic field is perpendicular to X-Z plane i.e. along y-axis. The externally applied fields $H_{O Y I G}$ and $H_{0 N I}$ are applied along zdirection in the longitudinal magnetization case and along $\mathrm{y}$-axis in transverse magnetization case, i.e., the magnetic fields $H_{0 Y I G} \& H_{0 N I}$ are applied along YIG layer and perpendicular to NI ferrite layer respectively. The elements of ferrite permeability in the case of longitudinal magnetization case are given as [23-24]:

$\mu_{f}=\left(\begin{array}{ccc}\mu_{r} & j \mu_{k} & 0 \\ -j \mu_{k} & \mu_{r} & 0 \\ 0 & 0 & \mu_{0}\end{array}\right)$

where $\mu_{r}$ and $\mu_{k}$ are given by:

$\mu_{r}=1+\frac{\omega_{m}\left(\omega_{0}-j \alpha \omega\right)}{\left(\omega_{0}-j \alpha \omega\right)^{2}-\omega^{2}}$

$\mu_{k}=\frac{\omega_{m} \omega}{\left(\omega_{0}-j \alpha \omega\right)^{2}-\omega^{2}}$

Here $\alpha$ is damping constant, $\omega_{0}\left(=2 \pi \gamma H_{0}\right)$ is resonance frequency with the gyro-magnetic ratio $(\gamma), H_{0}$ is the external magnetic field and $\omega_{m}\left(=2 \pi \gamma M_{s}\right)$ is the circular frequency with saturation magnetization $\left(M_{s}\right)$. Using transfer matrix method described in the [20], we can obtain a matrix which relates tangential components of electric and magnetic fields at one interface to subsequent interface of sub-layer of YIG ferrite in the unit cell as:

$$
\left(\begin{array}{l}
E_{I} \\
H_{I}
\end{array}\right)=M_{1}\left(\begin{array}{l}
E_{I I} \\
H_{I I}
\end{array}\right)
$$

The elements of $M_{1}$ for YIG sub-layer are:

$M_{1}=\left(\begin{array}{cc}\cos \left(k_{1 z} a\right) & j \eta_{1} \sin \left(k_{1 z} a\right) \\ \left(1 / j \eta_{1}\right) \sin \left(k_{1 z} a\right) & \cos \left(k_{1 z} a\right)\end{array}\right)$

Where $\eta_{1}=k_{1 z} / \omega \varepsilon_{0} \varepsilon_{f Y I G}, k_{1 z}=(\omega / c) \times \sqrt{\left[\left(n_{f Y I G}{ }^{2}\right)-(\beta)^{2}\right]}, k_{1 x}=(\omega / c) \times \beta$ and $n_{f Y I G}=$ $\sqrt{\mu_{r Y I G} \varepsilon_{f Y I G}}$. 
We can also express ferrite permeability for transverse magnetization case [23-24]. We obtain transfer matrix elements for the case of transverse magnetization configuration for NI ferrite sublayer of unit cell for TM mode [21] as follows:

$M_{2}=\left(\begin{array}{cc}\cos \left(k_{2 z} b\right) & j \eta_{2} \sin \left(k_{2 z} b\right) \\ \left(1 / j \eta_{2}\right) \sin \left(k_{2 z} b\right) & \cos \left(k_{2 z} b\right)\end{array}\right)$

$\eta_{2}=k_{2 z} / \omega \varepsilon_{0} \varepsilon_{f N I}, k_{2 z}=(\omega / c) \times \sqrt{\left[\left(n_{f N I}^{2}\right)-(\beta)^{2}\right]}, k_{2 x}=(\omega / c) \times \beta$ and $n_{f N I}=$

$\sqrt{\mu_{0} \varepsilon_{f N I}}$. The transfer matrix elements of the unit cell can be obtained by multiplying $M_{1}$ and $M_{2}$ as $M=M_{1} M_{2}$. By extracting the elements of $\mathrm{M}$, the dispersion relation can be obtained type-

1 1-D FPhCs as:

$\cos (K d)=\cos \left(k_{1 z} a\right) \cos \left(k_{2 z} b\right)-\frac{1}{2}\left[\frac{k_{1 z} \varepsilon_{f N I}}{k_{2 z} \varepsilon_{f Y I G}}+\frac{k_{2 z} \varepsilon_{f Y I G}}{k_{1 z} \varepsilon_{f N I}}\right] \sin \left(k_{1 z} a\right) \sin \left(k_{2 z} b\right)$

Where $K$ is Bloch wave number. Similarly, we can obtain dispersion relation for type-2 1-D FPhCs. The phase index of the considered structure is computed using the following expression:

$\phi=c K / \omega$

\section{Results and Discussion:}

In the article, we have numerically investigated the dispersion properties and phase index of type-1 \& type-2 magnetized 1-D FPhCs using equations (5) and (6). The thicknesses of YIG and NI layers are $a=\left(f \lambda_{0} / 4 n_{f Y I G}\right)$ and $b=(2-f) \lambda_{0} / 4 n_{f N I}$ where $f$ is the filling factor, $\lambda_{0}=$ $\left(2 \pi c / \omega_{m Y I G}\right), \omega_{m Y I G}=2 \pi f_{m Y I G}, f_{m Y I G}=\left(2.8 \times 10^{6} \times M_{S Y I G}\right)$. The resonance frequencies corresponding to YIG and NI ferrites are given by $\omega_{01 Y I G}=2 \pi f_{01 Y I G}, f_{01 Y I G}=(2.8 \times$ $10^{6} \times H_{01 Y I G}, \omega_{02 N I}=2 \pi f_{02 N I}, f_{01 N I}=\left(2.8 \times 10^{6} \times H_{0 N I}\right)$ respectively. The circular frequency corresponding to NI layer is $\omega_{m N I}=2 \pi f_{m N I}, f_{m N I}=\left(2.8 \times 10^{6} \times M_{s N I}\right)$ with saturation magnetization $M_{S N I}$. Normalized parallel wave vector, $\beta=k_{1 x} /(\omega / c)$ is same for the YIG and NI ferrite layer. The dielectric constant, saturation magnetization $\left(4 \pi M_{s}\right)$ and damping constant $(\alpha)$ of YIG are 15.0, $1780 O e$ and 0.0002 and for Ni ferrites are 9.0, $500 O e$ and 0.0008 respectively [23]. The trans-tech numbers of YIG ferrite and NI ferrite are G-113 and TT2-113 respectively [23]. In the paper, Oe denotes Oersted. For type-2 1D FPhCs structure $a=$ $\left(f \lambda_{0} / 4 n_{f N I}\right)$ and $b=(2-f) \lambda_{0} / 4 n_{f Y I G}$. The value of $\lambda_{0}$ is same for type-1 and type-2 1-D FPhCs structure.

\subsection{Effect of magnetic fields on the PBGs:}

To observe the effect of magnetic fields on the band structure with different $\beta$ at quarter wave staking $(f=1)$, Figs. $3 \& 4$ are plotted. Fig. 3 shows the variation of photonic band gaps (PBGs) with $H_{O Y I G}$ for type-1 magnetized 1-D FPhCs structure. At $\beta=1.1$ (black), with increases in $\mathrm{H}_{0 \mathrm{YIG}}$, frequency of $1^{\text {st }}$ PBGs is shifted towards higher frequency domain. The width of $2^{\text {nd }}$ PBGs increases with increases in $\mathrm{H}_{0 \mathrm{YIG}}$ and frequency domain remains nearly constant. Similar nature 
is observed for $\beta=2.1$ (red). For $\beta=2.8$ (green), new PBGs occur between $\omega \sim 0$ to $1 \times 10^{10}$ rad./sec. Also overlapping of PBG occurs in $1^{\text {st }}$ PBG. For $2^{\text {nd }}$ PBG, nature is contradictory as compared to $\beta(=1.1 \& 2.1)$. It is also found that width and frequency domain of PBGs remain nearly constant with increase in $\mathrm{H}_{0 \mathrm{NI}}$ (not shown here).

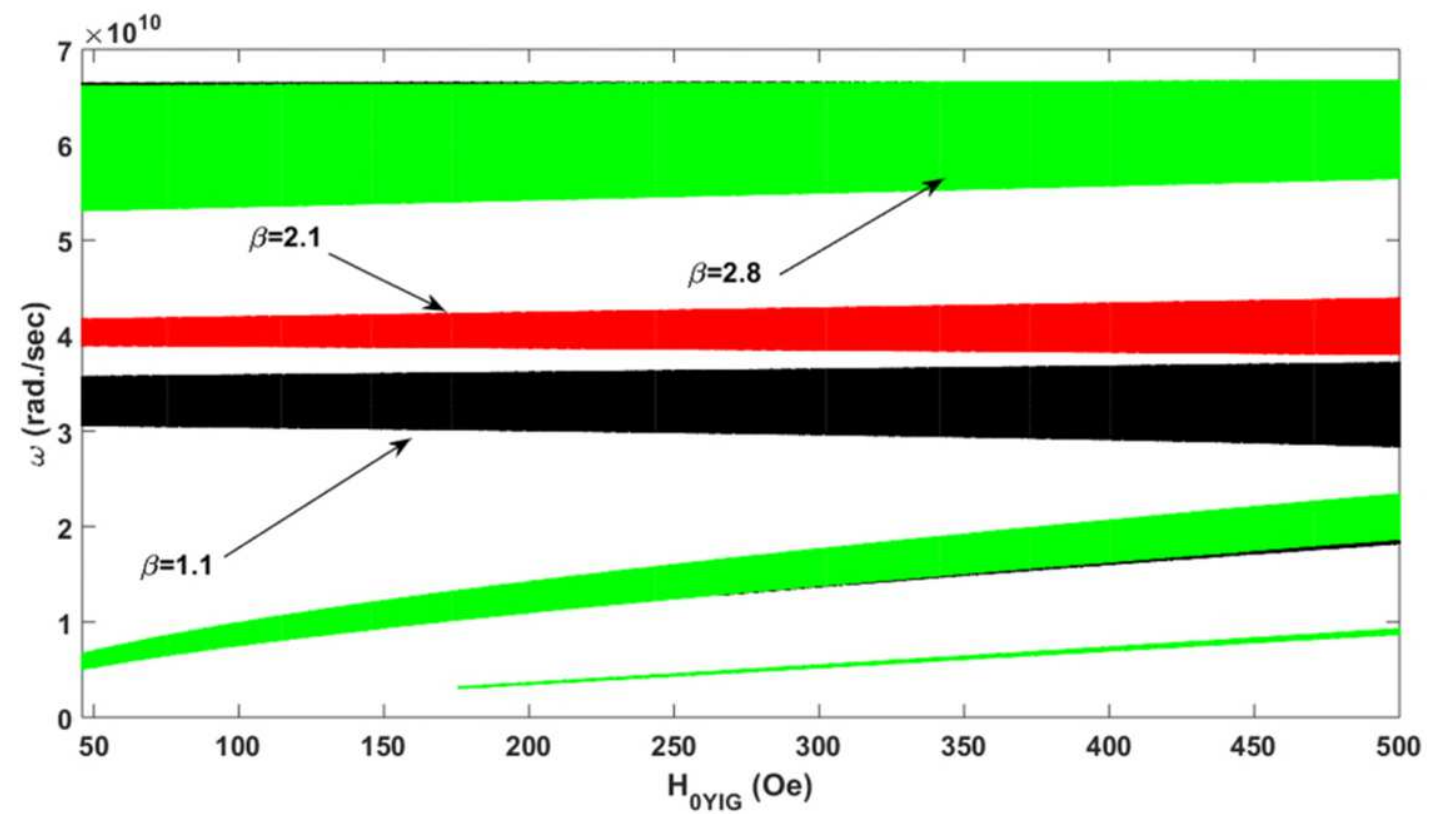

Fig. 3 Effect of magnetic field $\mathrm{H}_{0 \mathrm{YIG}}$ on PBGs for various $\beta(=1.1,2.1 \& 2.8)$ at fixed $\mathrm{H}_{0 \mathrm{NI}}=155$ Oe at $f(=1)$ in type-1 1-D FPhCs

For type-2 1-D FPhCs, fig. 4 is plotted. It is found that for $\beta=2.8$ (green), zero order PBGs is overlapped with $1^{\text {st }}$ order PBG and PBG is smaller as compared to $1^{\text {st }} \mathrm{PBG}$ in type-1 1-D FPhCs. For $\beta=1.1 \& 2.1$ width of PBG is small. The frequency domain of $1^{\text {st }} \mathrm{PBG}$ is shifted towards higher frequency domain with increase in $\mathrm{H}_{0 \mathrm{NI}}$. In this case also, there is no effect of transverse magnetic field $\mathrm{H}_{O Y I G}$ on the thickness \& frequency domain of PBG (not shown).

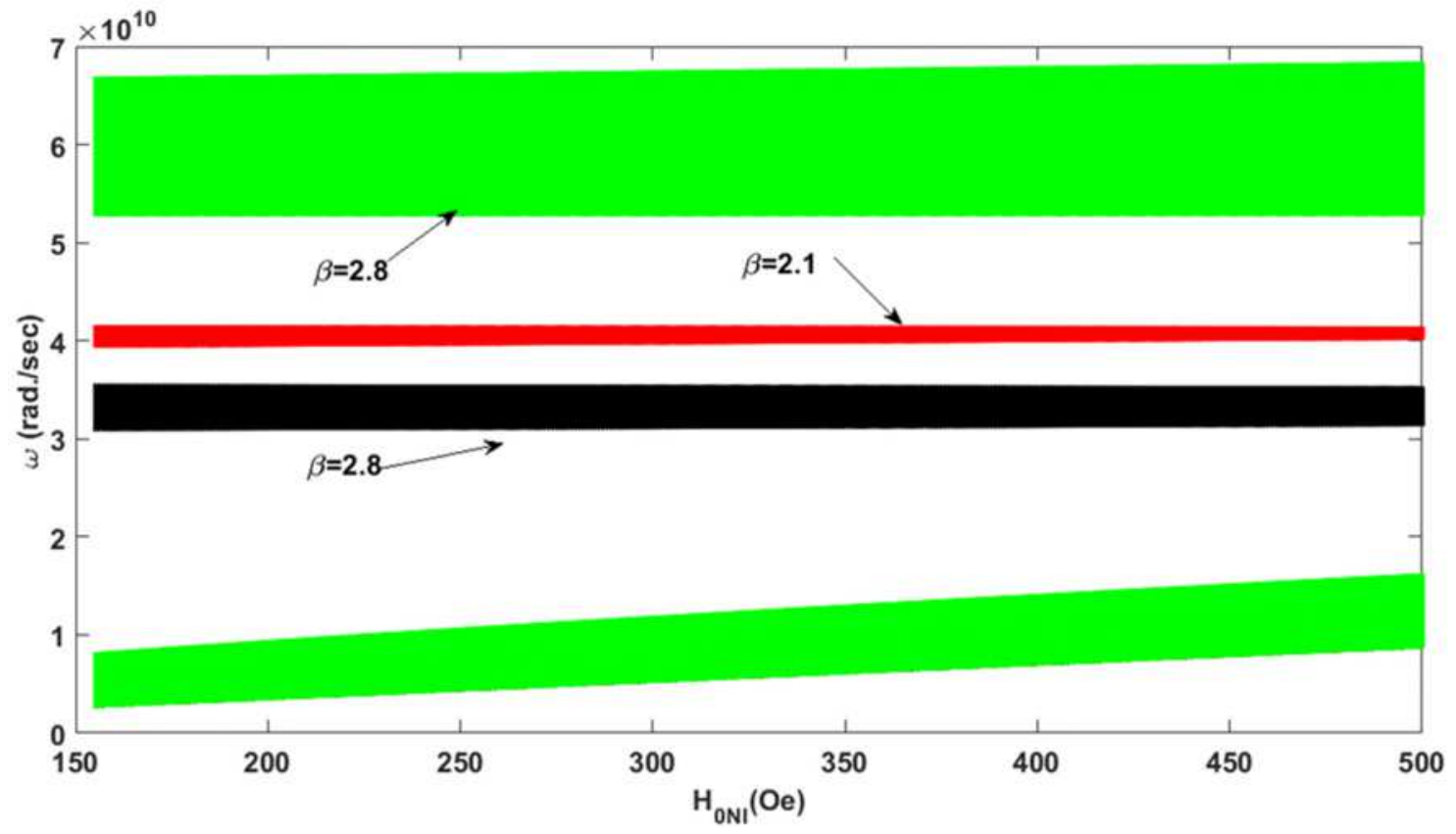

Fig. 4 Effect of magnetic field $\mathrm{H}_{0 \mathrm{NI}}$ on PBGs for $\beta(=1.1,2.1,2.8)$ at fixed $\mathrm{H}_{0 \mathrm{YIG}}=46$ Oe at $f(=1)$ for type-2 1-D FPhCs 


\subsection{Effect of normalized parallel wave vector $(\beta)$ on the dispersion curves}

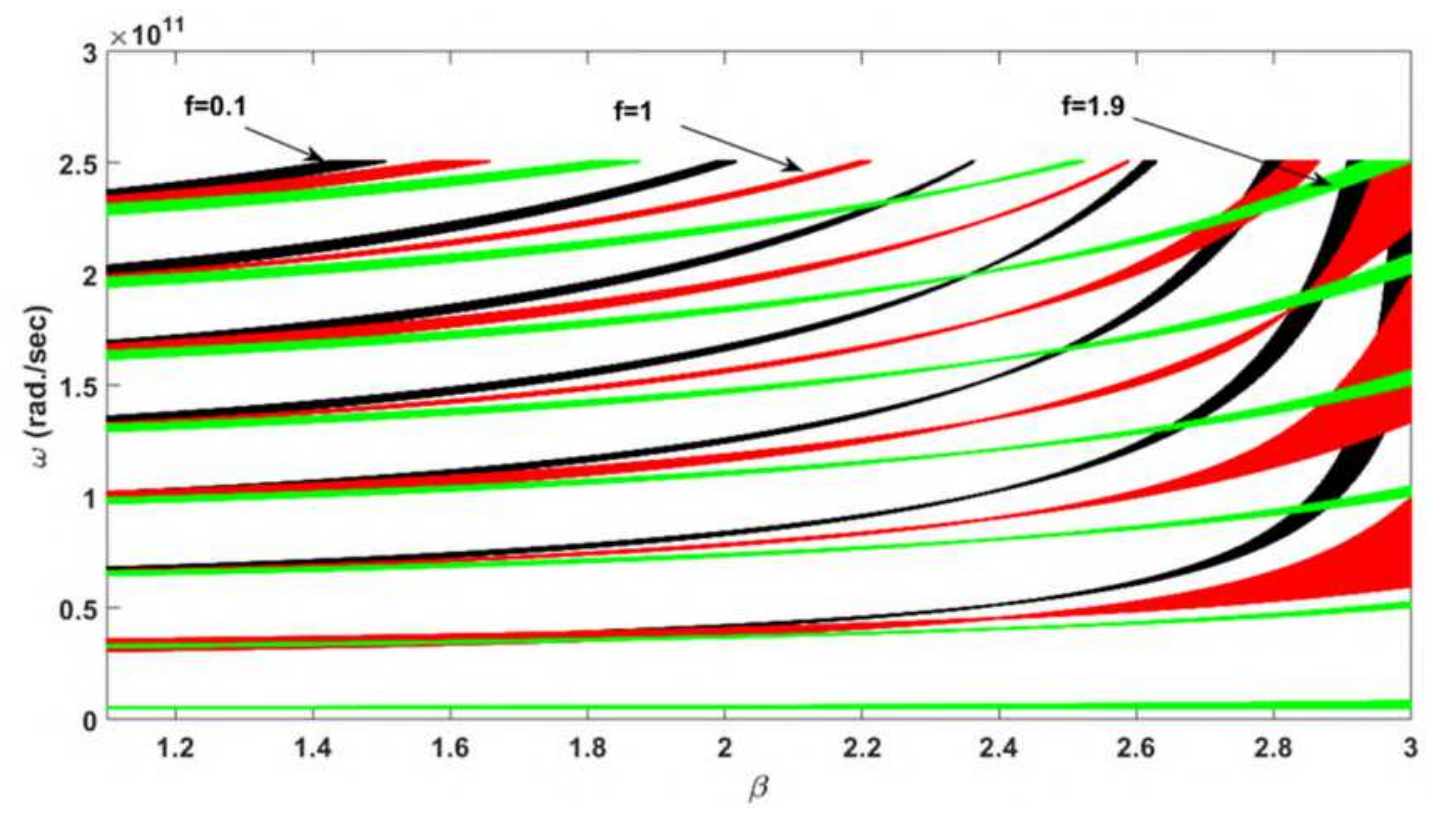

Fig. 5 Variation of $\omega$ with $\beta$ at $f=(0.1,1 \& 1.9)$ for type-1 1-D FPhCs

Fig.5 shows variation of $\omega \& \beta$ at different values of $f$ for $H_{O Y I G}=460 e \& H_{0 N I}=1550 e$ for type-1 1-D FPhCs. At $f=1$ (red), frequency domain of PBG is shifted towards higher frequency domain with increases in $\beta$. Also width of PBG decreases with increases in $\beta$. After $\beta \approx 2.38$ (Brewster angle), PBGs thickness increases. For, $f=0.1 \& 1.9$, PBGs shows nearly similar nature. At fixed $\beta$, PBGs is shifted to higher frequency with decreases in $\mathrm{f}$-value. The slope of $\omega-\beta$ curve decreases with increase in filling factor. So, for thicker YIG layer, Phase velocity $(\omega / \beta)$ along interface decreases. Here Zeroth order PBGs occur correspondingly to $f=1.9$ (green) only.

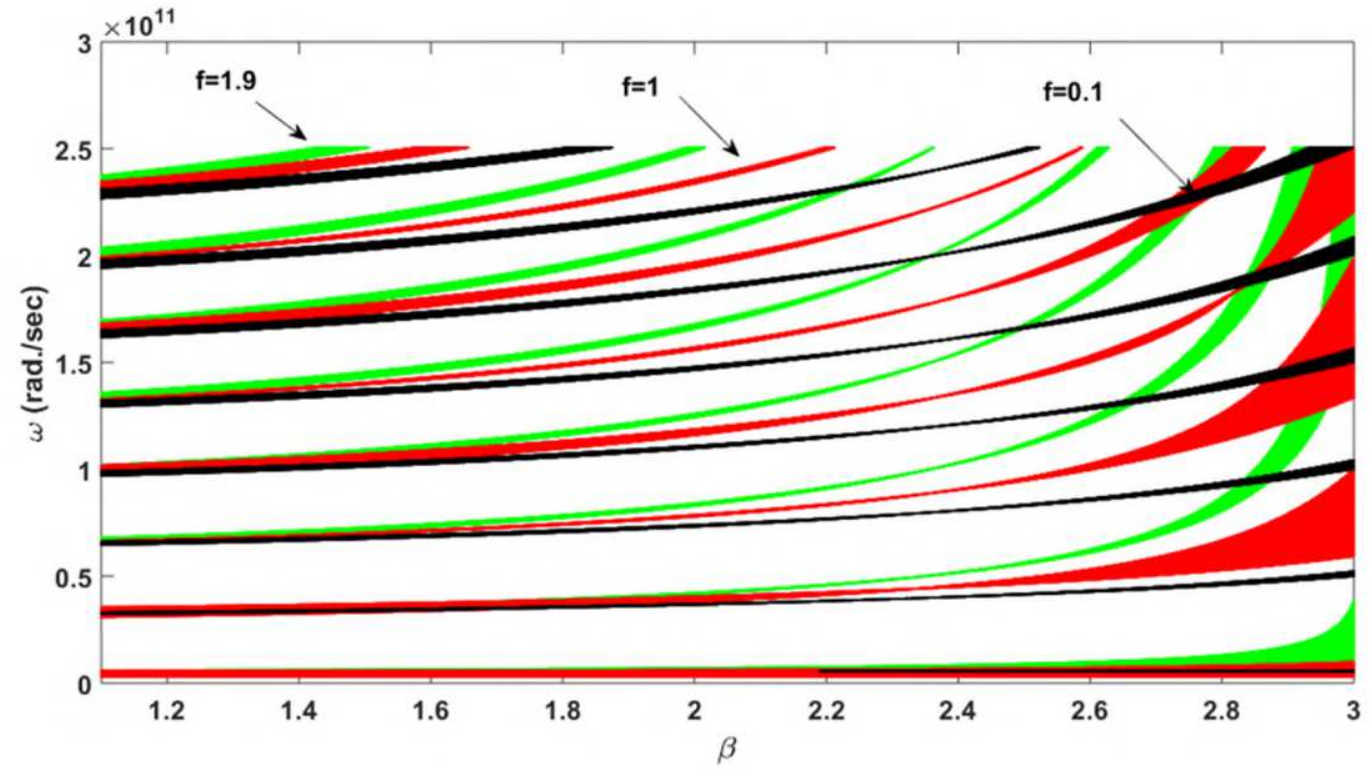

Fig. 6 Variation of $\omega$ with $\beta$ at $f(=0.1,1 \& 1.9)$ for type-2 1-D FPhCs 
Here fig.6 describes relation of $\omega-\beta$ at different ' $f$ ' for type-2 1-D FPhCs. PBG variation with $\beta$ for quarter wave stacking is similar as type-1 1-D FPhCs. But for $f=0.1 \& 1.9$, the frequency domain of PBG are opposite as for type-1 1-D FPhCs. The reason is that here with increase in $f$ value, YIG layer becomes thinner and width of NI layer increases.

\subsection{Effect of filling factor on the dispersion curves}

Effect of filling factor ' $f$ ' on PBGs for type-1 1-D FPhCs is shown in fig.7. Here PBGs occurs in the forms of lobes. At $\beta=1.1$, for $1^{\text {st }}$ order PBG, thickness of PBG increases up to $f=1$ (width of PBG is maximum here). There after its width increases in filling factor. Number of lobes increases with increases in order of PBGs. Similar nature is observed for $\beta=2.1$ (red) $\& \beta=$ 2.8 (green). Also, all order PBGs becomes thicker as compared to $\beta=1.1 \& 2.1$. The slope of $\omega \&$ $f$ decreases with increase in $\beta$-value.

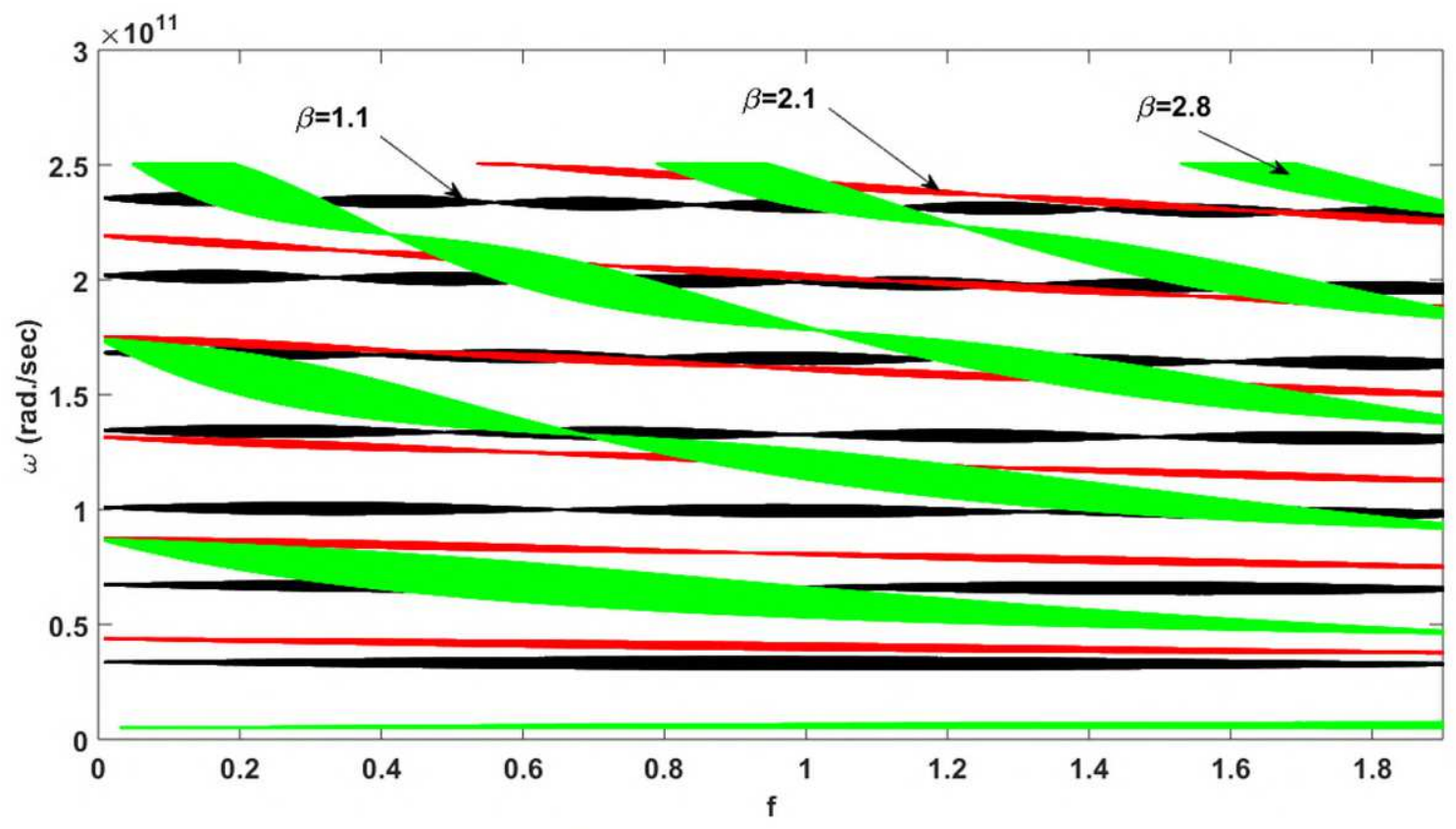

Fig. 7 Variation of $\varrho$ with $f$ at $\beta=1.1,2.1 \& 2.8$ for type-1 1-D FPhCs

For type-2 1-D FPhCs, Fig. 8 is plotted. Here also, PBGs occur in the form of lobes. Simillar nature is observed but the slope of $\omega-f$ curves is increased with increase in $\beta$-value. The reason for this opposite nature as that of type-1 magnetized 1-D FPhCs is that with increase in $\mathrm{f}$ value, the NI layer becomes thicker and thickness of YIG layer decreases. In type-1 magnetized 1-D FPhCs structure, the width of YI layer is increased with increase in $f$-value. 


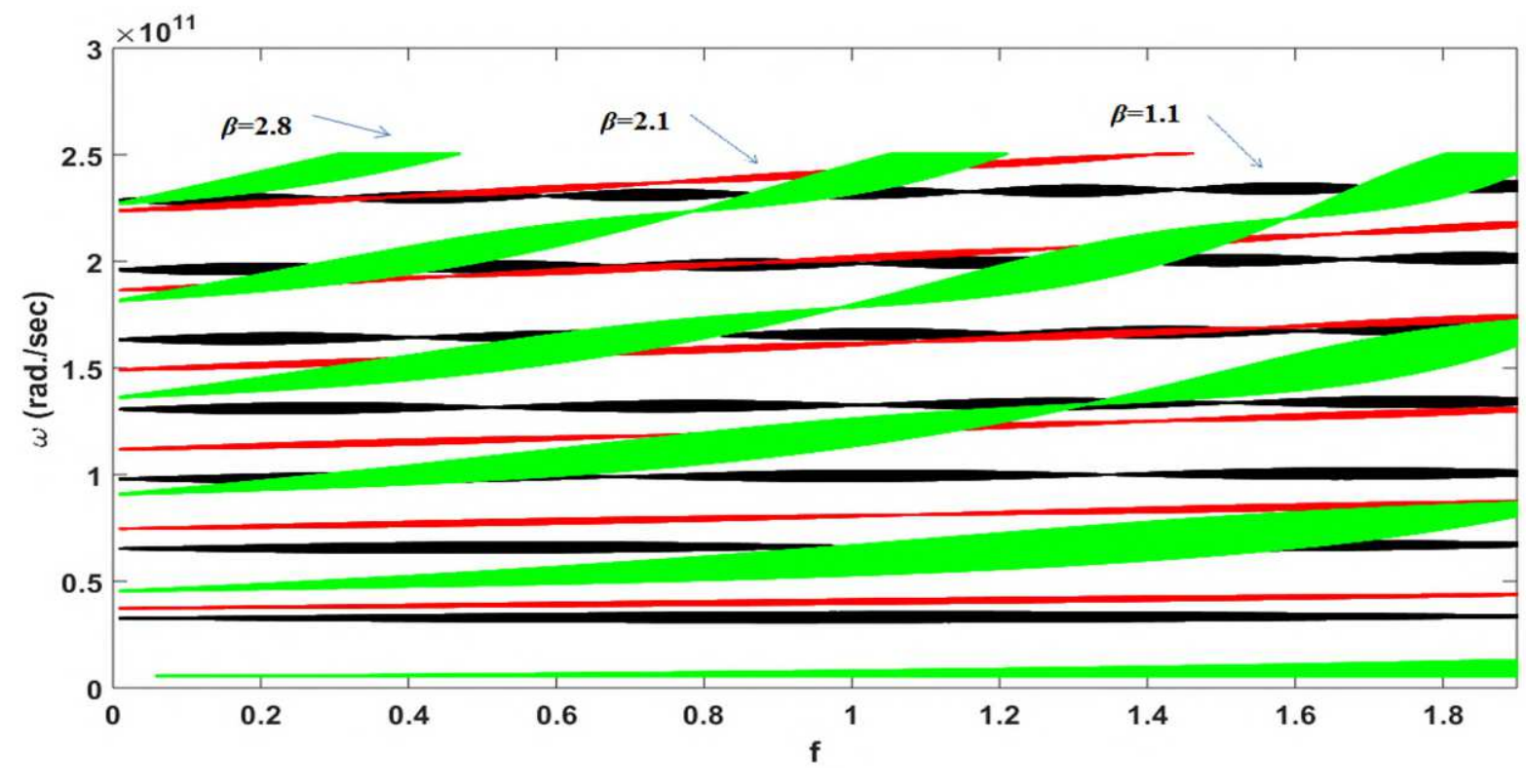

Fig. 8

Variation of $\omega$ with $f$ at $\beta=1.1,2.1 \& 2.8$ for type-2 1-D FPhCs

\subsection{Variation in phase index $(\phi)$ with the filling factor at $\beta=(1.1,2.1 \& 3.1)$}

The phase index of the considered structures is computed using equation (6). Figs. 9 and 10 show the phase index variation with filling factor at $H_{0 Y I G}=46 O e$ and $H_{0 N I}=1550 e$ at different $\beta$ for type-1 \& type-2 1-D FPhCs structures. In Fig. 9, at $\beta=1.1$, PBGs in phase index occurs in the form of lobes. Also, with increase in $f$-value, magnitude of $\phi$ increases. As we move towards lower $\phi$-value, number of lobe increases. Similar nature is observed for other $\beta$ values. Zero $\phi$ value is accessible to EM waves in considered frequency range at $\beta=1.1,2.1 \& 2.8$ at all $f$ value.

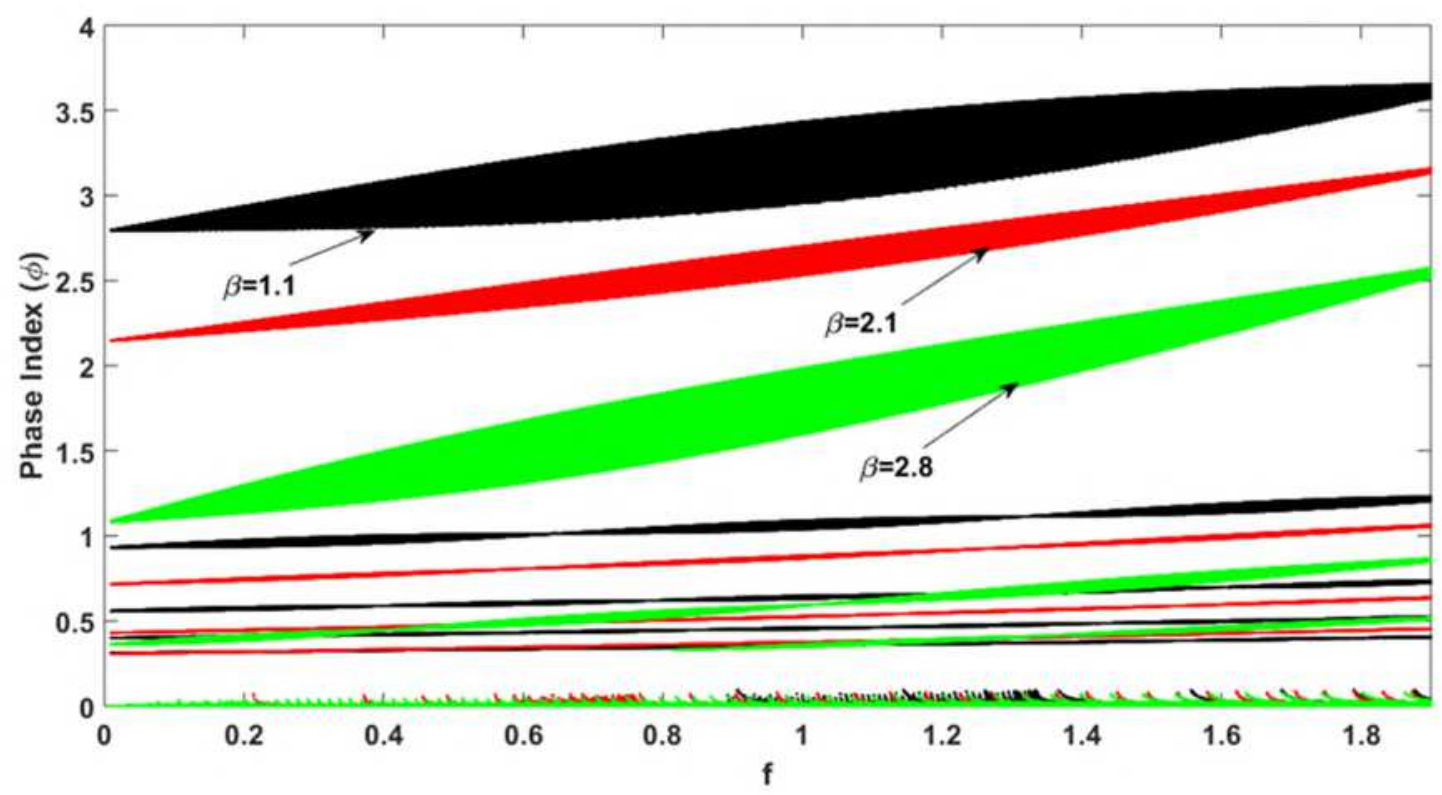

Fig. 9 Variation of $\phi$ with $f$ at $\beta=(1.1,2.1 \& 2.8)$ for type-1 1-D FPhCs 
Fig.10 is plotted to show the variation of $\phi$ with $f$ at $\beta(=1.1,2.1 \& 2.8)$ for type-2 FPhCs. The PBGs in phase index also occur in the form of lobes. But the slope of $\phi$ with $f$-value is opposite to type-1 1-D FPhCs structure. The reason is that with increase in $f$-value, NI layer becomes thicker whereas in type-11-D FPhCs structure, NI becomes thinner.

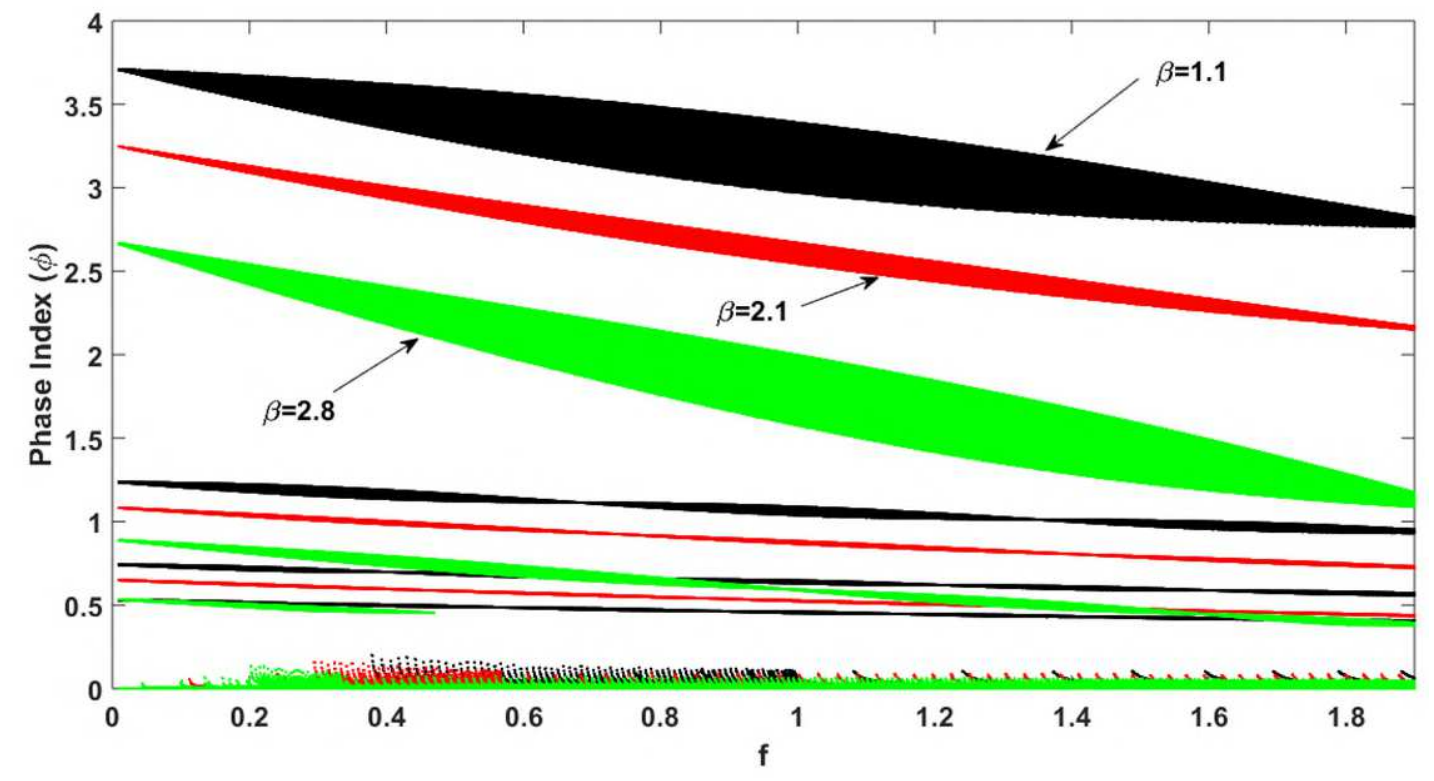

Fig. 10 Variation of $\phi$ with $f$ at $\beta=1.1,2.1 \& 2.8$ for type-2 1-D FPhCs

\subsection{Variation in phase index $(\phi)$ at $f=(0.1,1 \& 1.9)$ with $\beta$}

Figs. 11 is plotted to shows the variation of $\phi$ with $\beta$ at different $f(=0.1,1 \& 1.9)$ for type-1 1-D FPhCs structure.

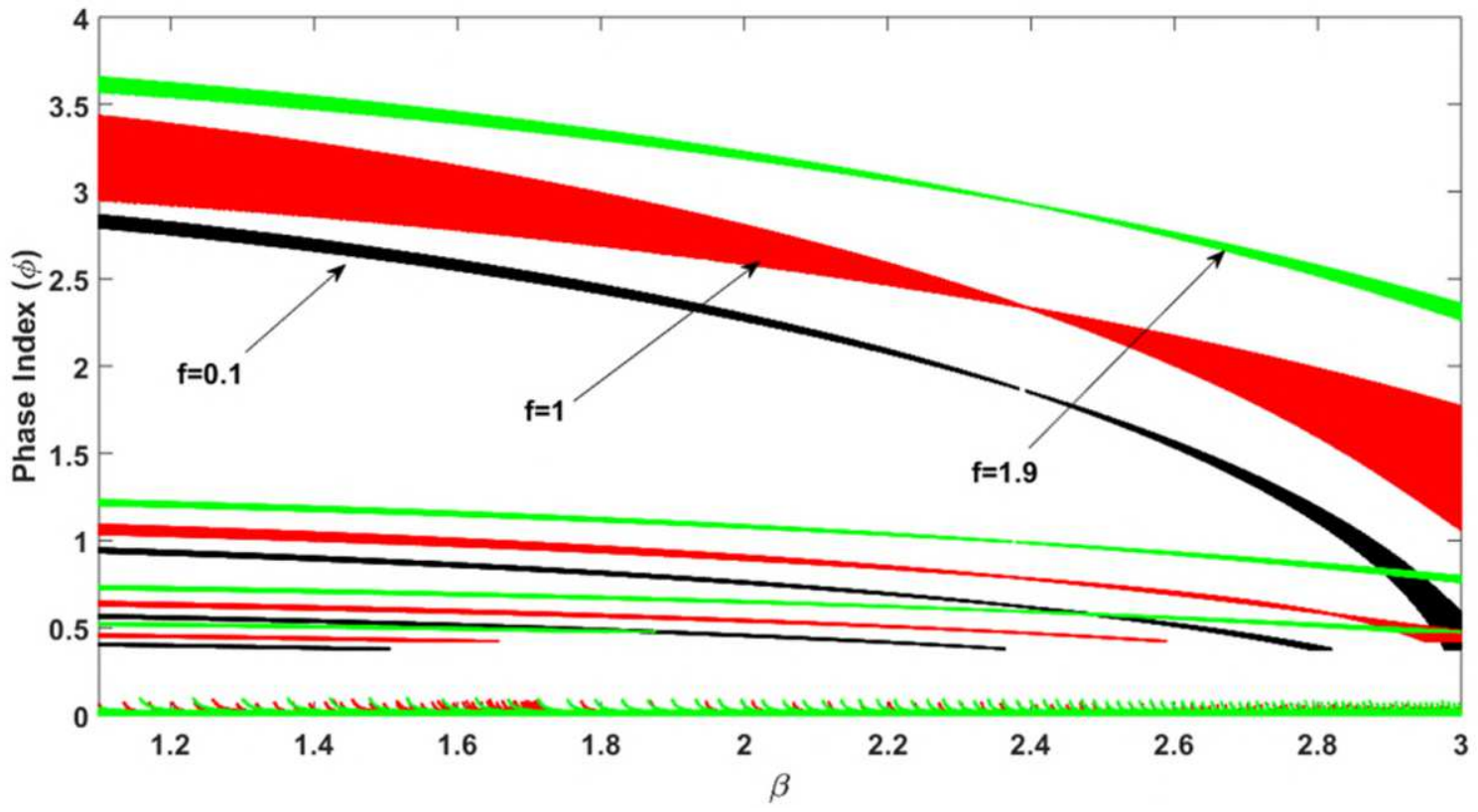

Fig. 11 Variation of $\phi$ with $\beta$ at $f=(0.1,1 \& 1.9)$ for type-1 1-D FPhCs 
At $\mathrm{f}=0.1$ (black), with increase in $\beta$, the thickness of PBGs in phase index is reduced. At $\beta=$ 2.38, flipping of PBGs occurs. Similar nature is observed for $\mathrm{f}=1$ (red) \& 1.9 (green). At quarter wave stacking $(f=1)$, the flipping of PBGs in phase index is clearly seen. Also, PBGs in phase is thicker as compared to $f=0.1 \& 1.9$.

Fig. 12 is also plotted for type-2 FPhCs with $\beta$ at different $f(=0.1,1.1 \& 1.9)$. If we compare figs. $(11 \& 12)$, it is observed that lower filling factor $(f=0.1)$ PBGs are shifted to upper side of $f=1$ case.

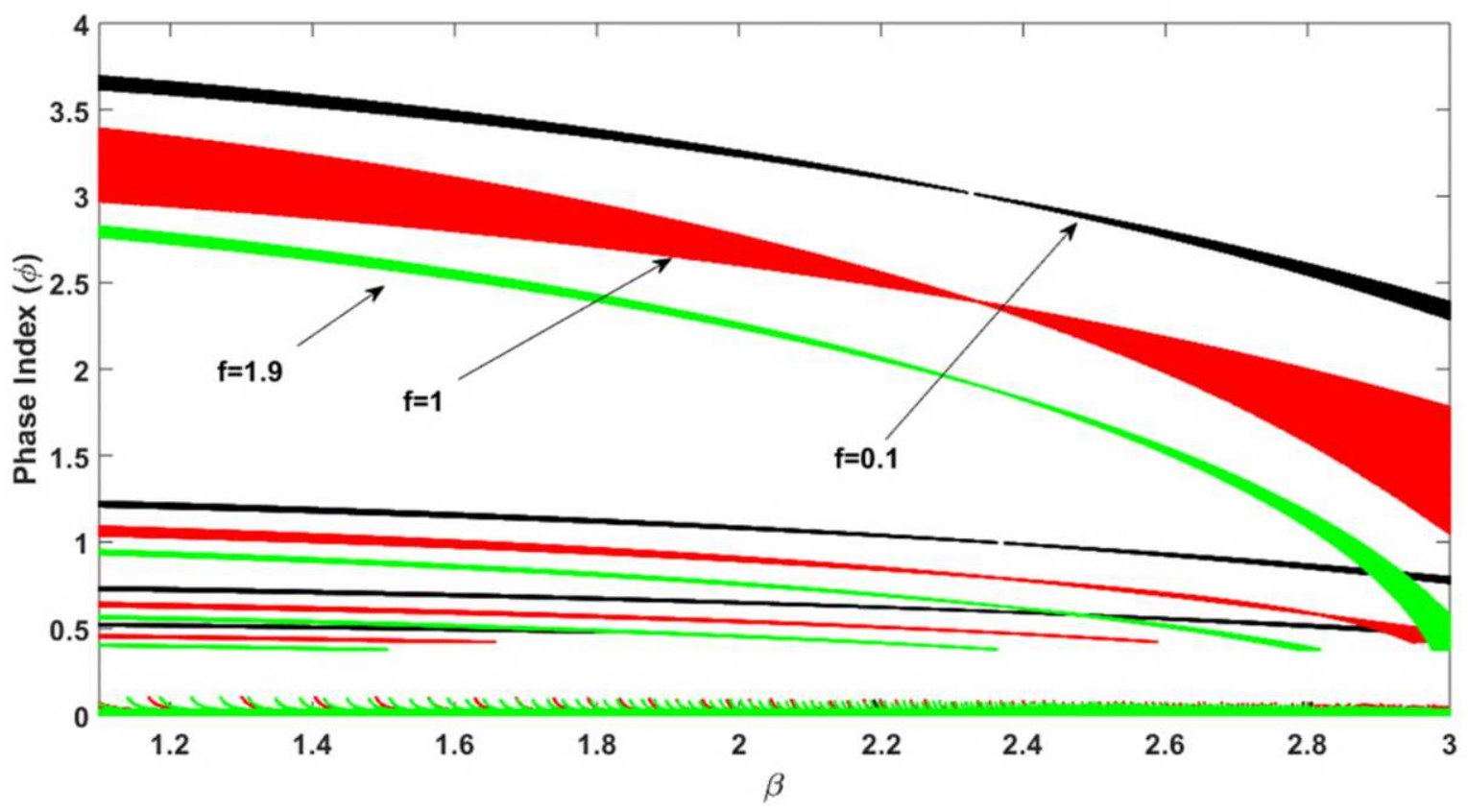

Fig. 12 Variation of $\phi$ with $\beta$ at $f=(0.1,1 \& 1.9)$ for type-2 1-D FPhCs

\subsection{Variation of phase index $(\phi)$ with external magnetic fields}

To make deep insight of phase index $\phi$ with external magnetic fields at different values of $\beta$, we have shown 3-d plots (Figs. $13 \&$ 14) for type-1 \& type-2 1-D FPhCs structures. These figures are plotted at quarter wave stacking condition. On x-axis, normalized frequency $\left(\omega / \omega_{\text {mYIG }}\right)$, on y-axis, $H_{0 Y I G}$ and on z-axis, phase index $\phi$ are displayed. In Fig. 13, it is observed that phase index $\phi$ is very sensitive function of $\omega, \beta$ and $H_{0 Y I G}$. In the lower frequency regime $\left(\omega / \omega_{\text {mYIG }} \cong\right.$ 0 ), overlapping of PBGs in phase index is observed. After that PBGs in phase index occur at different frequency at different magnitude for different values of $\beta$. At fixed $H_{0 Y I G}$, PBGs in phase index is shifted towards higher frequency domain when $\beta$ is increased from 1.1 (black) to 2.8 (green). It is interesting also that PBGs in phase index is reduced to zero at lower frequency 
range $\left(\omega / \omega_{m Y I G} \cong 0\right)$ and multiple PBGs are observed as peaks for 1.1 (black) to 2.8 (green) in the vicinity of $\omega / \omega_{m Y I G} \cong 0$. In this case $H_{0 N I}$ is taken as $155 \mathrm{Oe}$.

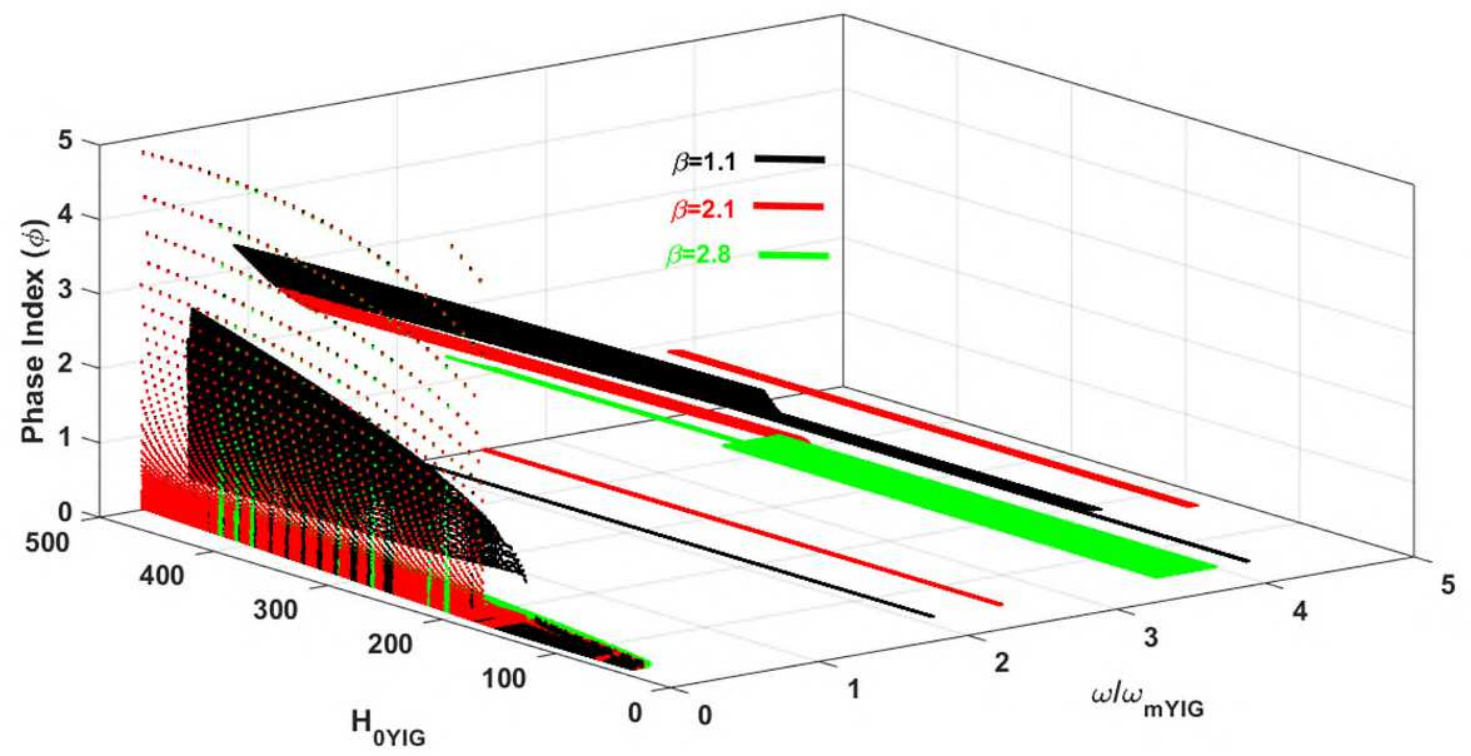

Fig. 13 Variation of $\boldsymbol{\phi}$ with $\mathbf{H}_{0 Y I G}$ at different $\boldsymbol{\beta}$ with the normalized frequency $\left(\omega / \omega_{m Y I G}\right)$ for type-1 1-D FPhCs

In type-2 1-D FPhCs structure (Fig. 14), these peaks in the lower frequency $\left(\omega / \omega_{\text {mYIG }} \cong 0\right)$ are clearly visible for $\beta=2.1$ (red).

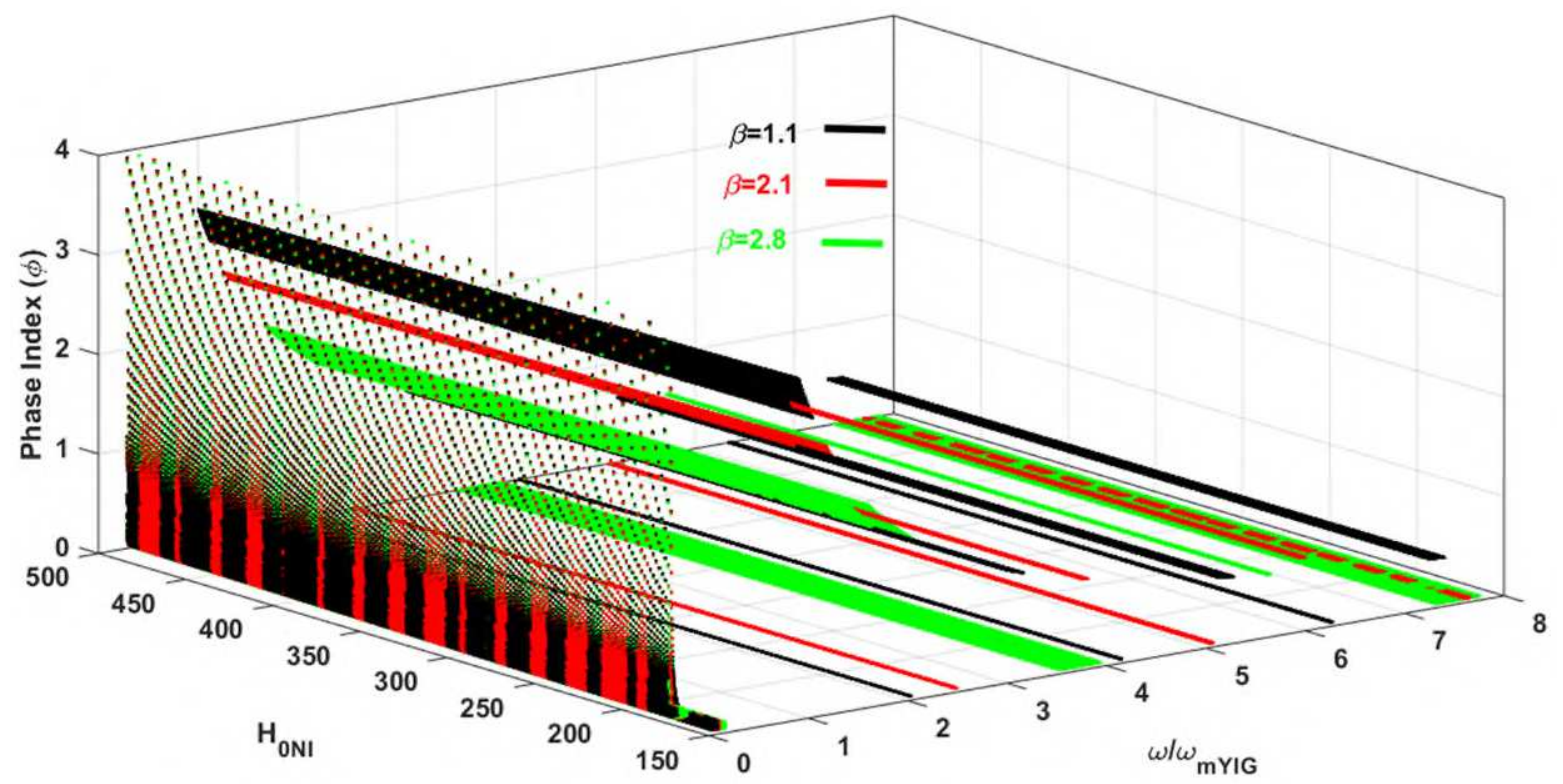

Fig. 14 Variation of $\phi$ with $\mathbf{H}_{0 \text { YIG }}$ at different $\beta$ with the normalized frequency $\left(\omega / \omega_{m Y I G}\right)$ for type-2 1-D FPhCs 


\section{Conclusions}

The present paper is devoted to study the effect of external fields, $\beta$ and filling factor on the dispersion characteristics and phase index of two types of 1-D FPhCs structure having mixed field configurations. In the $\left(\omega-H_{0}\right)$ diagrams, it is found that there is effect of longitudinal magnetic fields for both of types of 1-D FPhCs. With increase in longitudinal magnetic fields $\left(\mathrm{H}_{0 \text { YIG }}\right.$ for type $-1 \& \mathrm{H}_{0 \mathrm{NI}}$ for type -2$)$, the frequency domain of PBGs is shifted towards higher side. The $(\omega-\beta)$ curves show that for both types of 1-D FPhCs structures, at fixed $\mathrm{f}-$ value, PBGs are shifted towards higher frequency domain with increase in $\beta$. At fixed $\beta$, PBGs corresponding to thinner YIG ferrite layer are in higher frequency domain. Also, the slope of $\omega-\beta$ curve decreases with increase in filling factor. So, for thicker YIG layer, phase velocity $(\omega / \beta)$ along interface decreases. The variation of $(\omega-f)$ curve reveals that PBGs occur in the form of lobes for both types. For type-1 1-D FPhCs, at fixed $\beta$, slope of $\omega$ with $f$ decreases whereas for type-2, we obtain contrary result.

It is also observed that phase index is very sensitive to external magnetic fields, $\beta$ and filling factor. The $\phi-f$ curves at fixed $\beta$ for both types of 1-D FPhCs show that PBGs in phase index occurs in the form of lobes. As we move towards lower $\phi$-value, number of lobe increases. The pronounced flipping of PBGs in phase index is observed in $\phi-\beta$ curve at quarter wave stacking condition for both types. The $3 \mathrm{~d}$ plots of phase index, external magnetic field and frequency show that there are multiple peaks in the PBGs of phase index in the lower frequency $\left(\omega / \omega_{m Y I G} \cong 0\right)$ for both types of 1-D FPhCs structures.

\section{ACKNOWLEDGMENT}

Author, Yogesh Sharma is grateful to Dr. Vivek Singh and Dr. A. K. Singh for their continuous encouragement and support in many ways.

\section{Reference:}

1. M. Inoue, K. Arai, T. Fujii, M. Abe, J. Appl. Phys. 83, 6768 (1998)

2. M.J. Steel, M. Levy, R.M. Osgood Jr., IEEE. Photon. Technol. Lett. 12, 1171 (2000)

3. I. Bita, E.L. Thomas, J. Opt. Soc. Am. B. 22, 1199 (2005)

4. Z. Wang, S. Fan, Opt. Lett. 30, 1989 (2005)

5. R. Wolfe, R.A. Lieberman, V.J. Fratello, R.E. Scotti, N. Kopylov, Appl. Phys. Lett. 56, 426 (1990)

6. G. Mumcu, K. Sertel, J.L. Volakis, I. Vitebskiy A. Figotin, IEEE APS Symposium. 2, 1395 (2004)

7. A. Figotin, I. Vitebsky, Phys. Rev. E. 63, 066609 (2001)

8. A. Figotin, I. Vitebskiy, Phys. Rev. B. 67, 165210 (2003)

9. $\quad$ S. Y. Liu, W. L. Lu, Z.F. Lin, S.T. Chui, Appl. Phys. Lett. 97, 201113 (2010)

10. M. Vasiliev, K. Alameh, V. Belotelov, V.A. Kotov, A.K. Zvezdin, J. Lightwave Technol. 24, 2156 (2006) 
11. M. Zamani, and M. Ghanaatshoar, Opt. Eng. 54, 097103 (2015)

12. Z. Yu, Z. Wang, Appl. Phys. Lett. 90, 121133 (2007)

13. X. Xi, M. Lin, W. Qiu, Z. Ouyang, Q. Wang, Q. Liu, Scientific Report. 8, 1 (2018)

14. A.M. Grishin, S.I. Khartsev, J. Magn. Soc. Jpn. 32, 140 (2008)

15. J.-X. Liu, H.-Y. Xu, Z.-K. Yang, X. Xie, Y. Zhang, H.-W. Yang, Plasmonics. 12, 971 (2017)

16. K. Bi, K. Huang, L.Y. Zeng, M.H. Zhou, Q. M. Wang, Y. G. Wang, M. Lei, PLOS ONE. 10, 1 (2015)

17. F. Fan, S. -J. Chang, C. Niu, Yu Hou, X. -H. Wang, Opt. Commun. 285, 3763 (2012)

18. V.I. Fesenko, V.R. Tuz, I.V. Fedorin, H.-Bo Sun, V.M. Shulga, W. Han, J. Electromag. Wave. 31, 1265 (2017)

19. N. Dib, A. Omar, IEEE Trans. Microw. Theory Tech. 50, 1730 (2002)

20. Y. Sharma, and S. Prasad, Superlattice Microst. 120, 463 (2018)

21. Y. Sharma, and S. Prasad, Eur. Phys. J. D. 73, 166 (2018)

22. Y. Sharma, S. Prasad, J Supercond Nov Magn (2018). https://doi.org/10.1007/s10948-018-4904-4

23. D. M. Pozar, Microwave Engineering, 3rd ed. (Wiley publication New York, 2004)

24. M. Igarashi, Y. Naito, IEEE. Transactions on Magnetics. 13, 1664 (1997) 
Figures

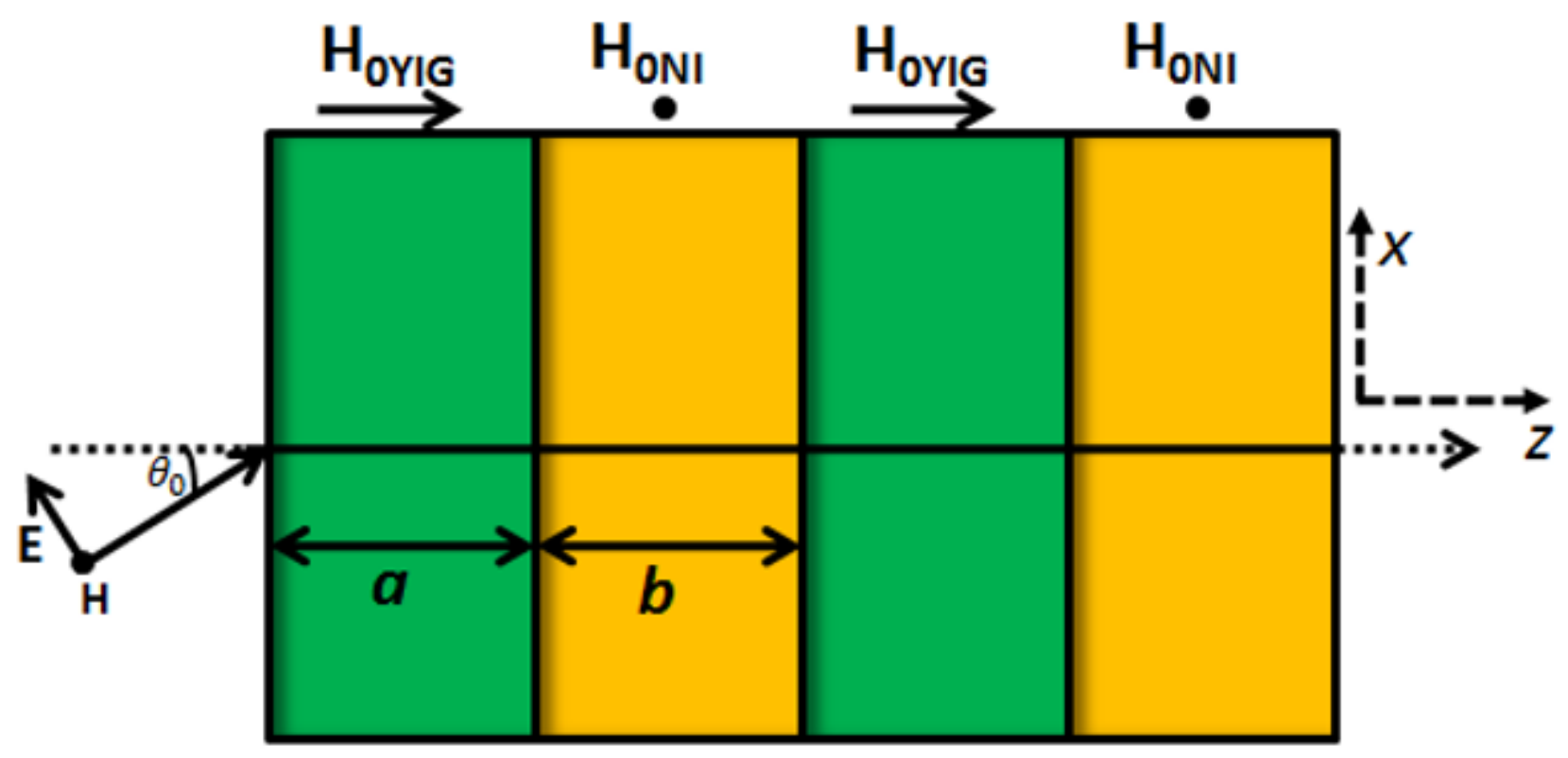

Figure 1

Schematic diagram of type-1 1-D FPhCs

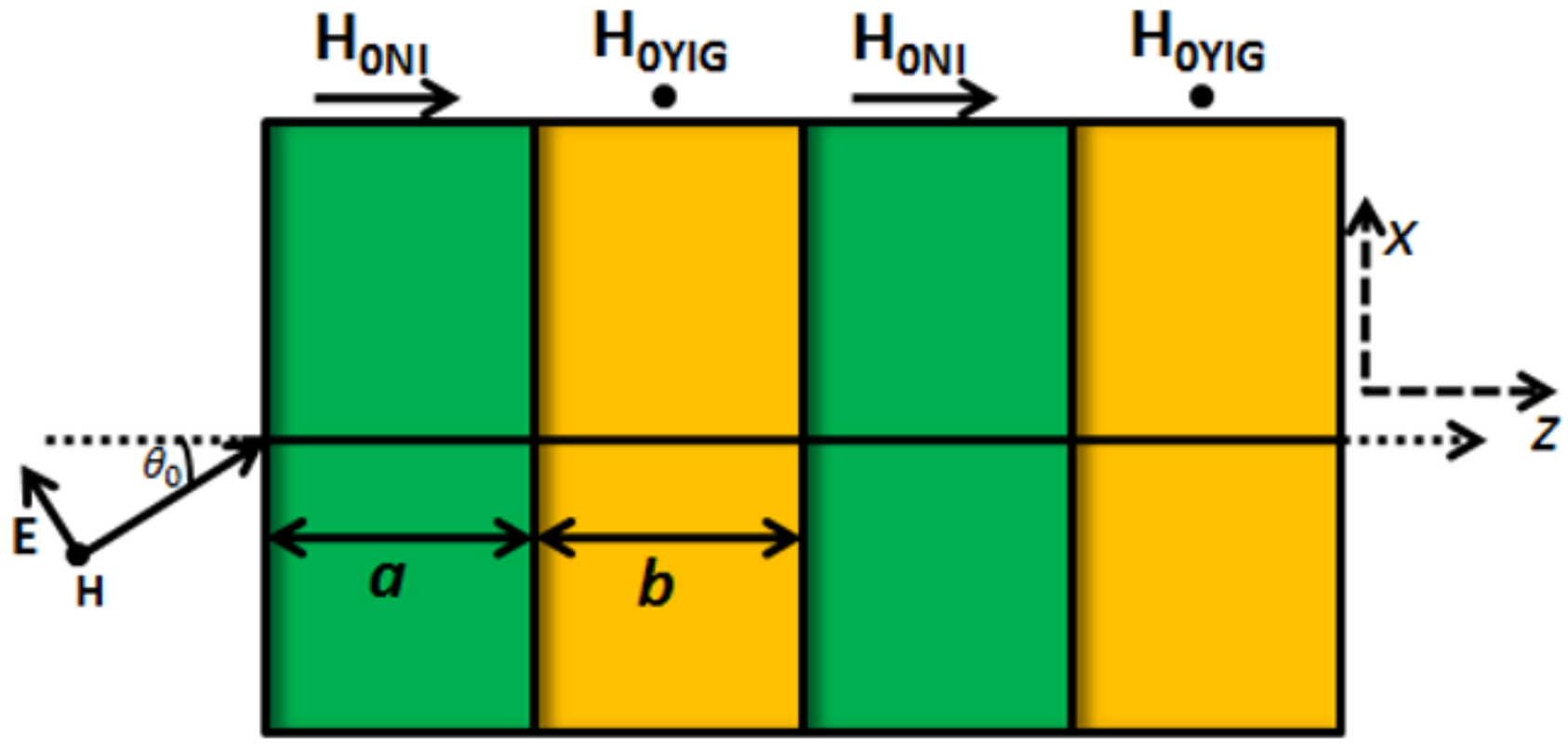

Figure 2

Schematic diagram of type-2 1-D FPhCs 


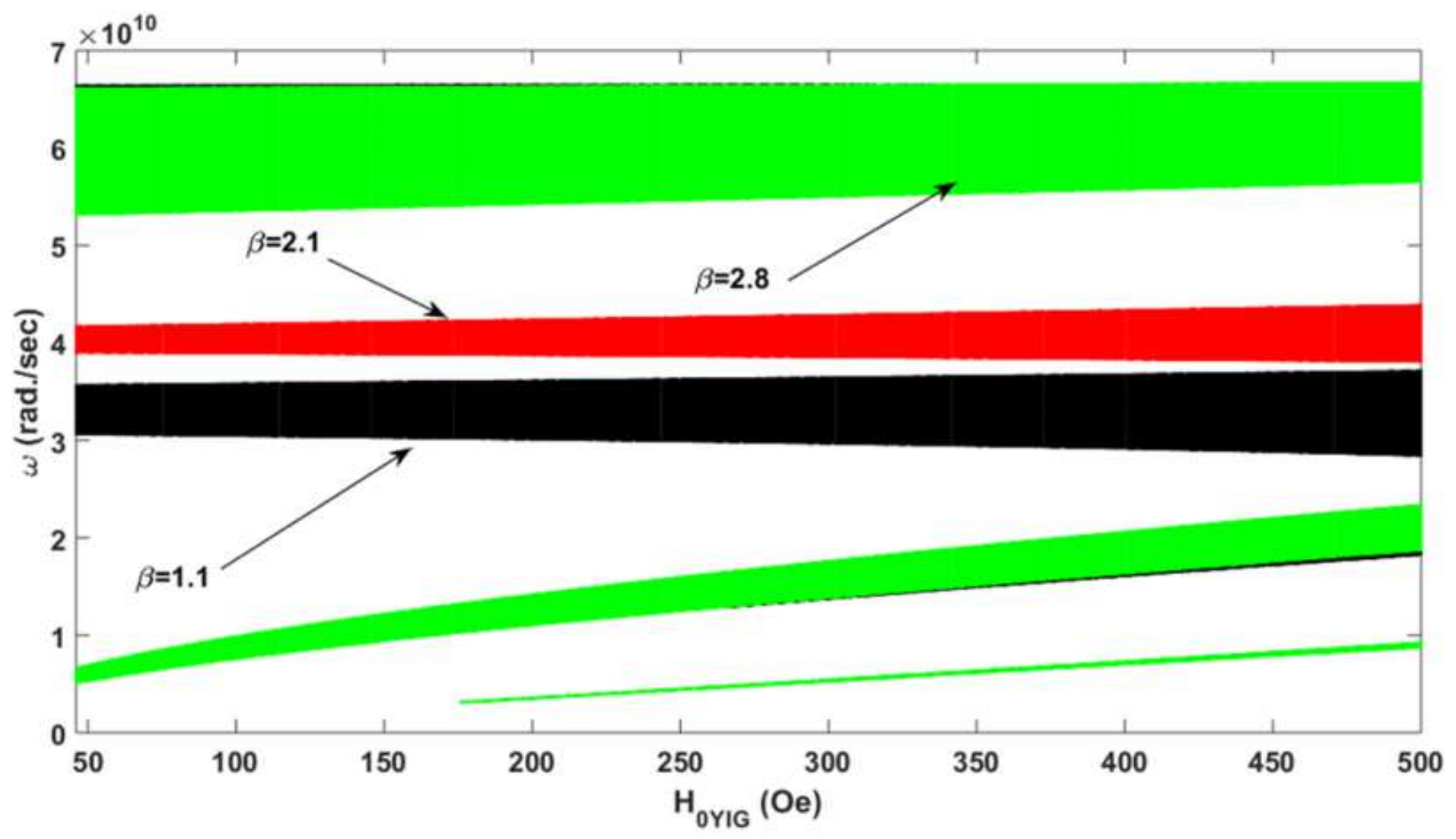

Figure 3

Effect of magnetic field HOYIG on PBGs for various $\beta(=1.1,2.1$ \& 2.8) at fixed $\mathrm{HONI}=155$ Oe at $f(=1)$ in type-1 1-D FPhCs

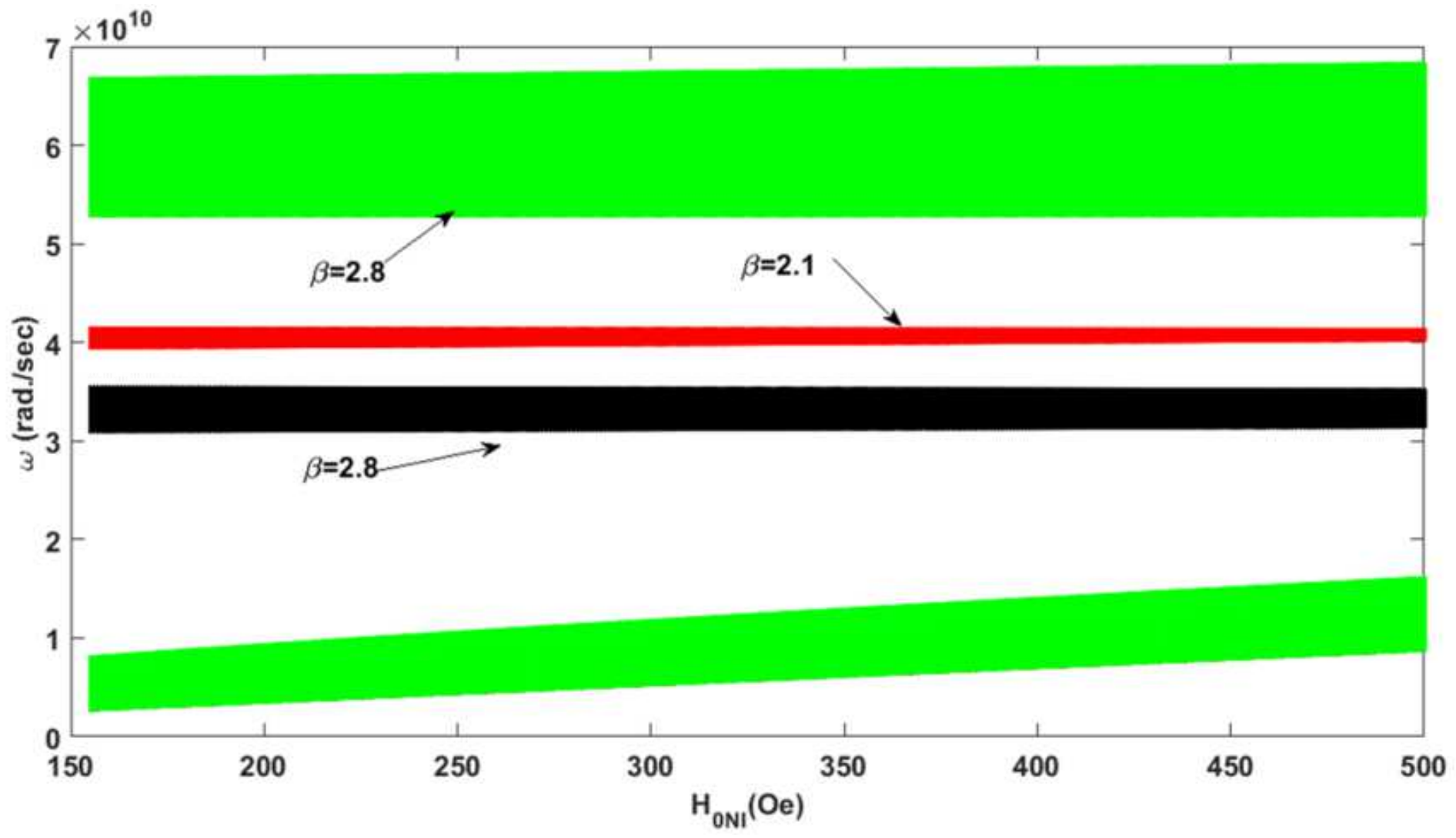


Figure 4

Effect of magnetic field HONI on PBGs for $\beta(=1.1,2.1,2.8)$ at fixed HOYIG $=46$ Oe at $f(=1)$ for type-2 1-D FPhCs

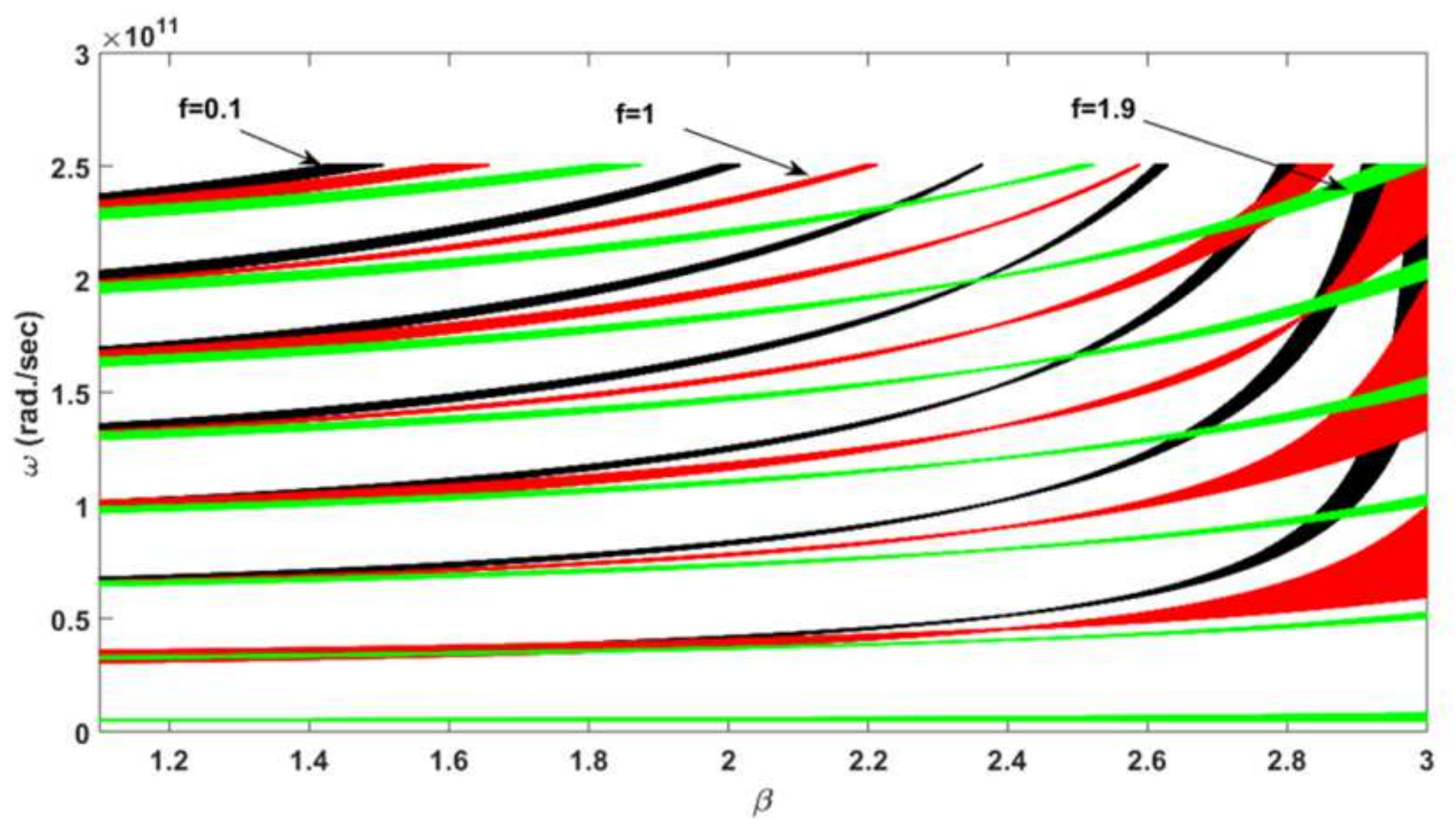

Figure 5

Variation of $\omega$ with $\beta$ at $f=(0.1,1 \& 1.9)$ for type-1 1-D FPhCs 


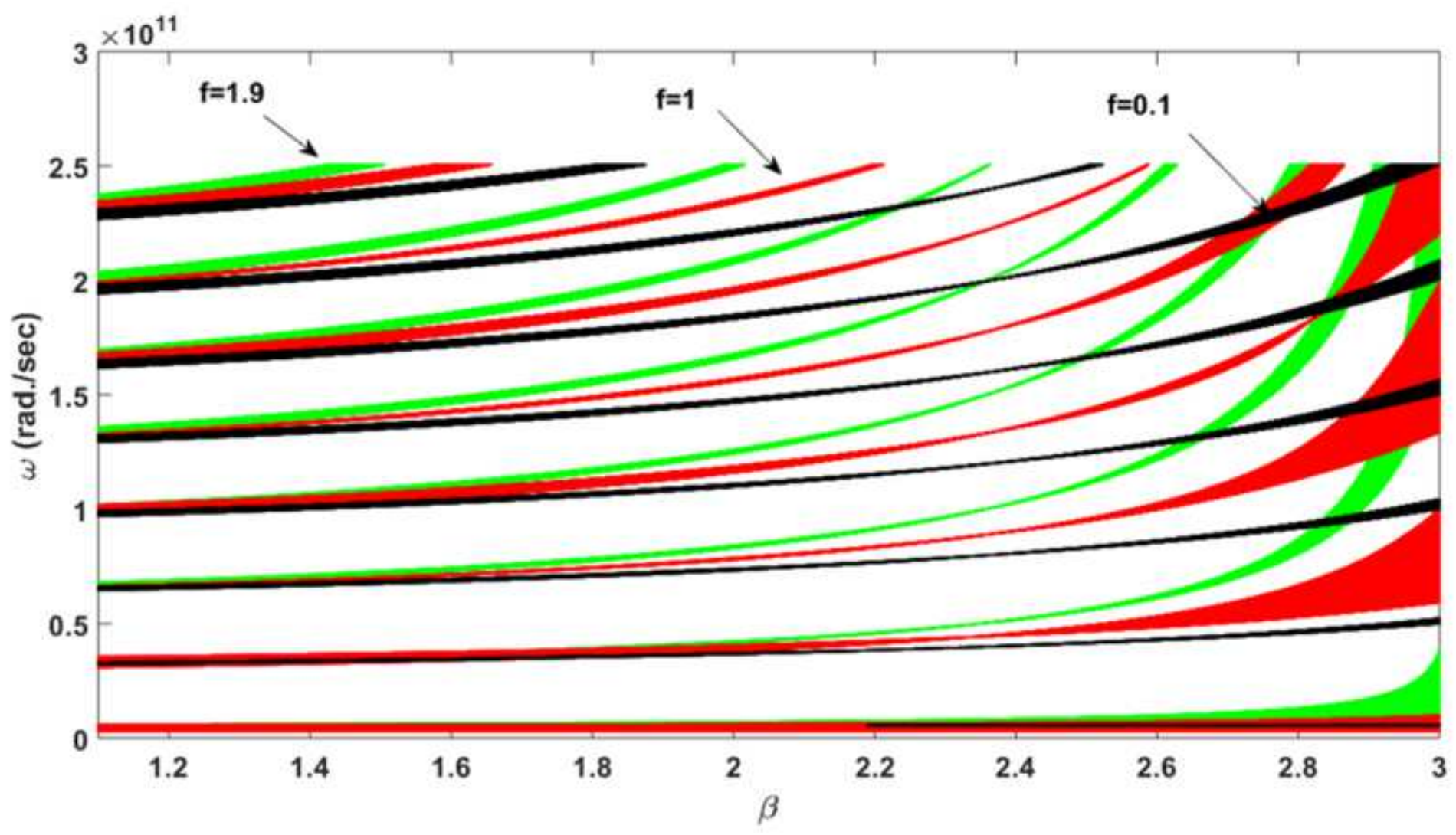

Figure 6

Variation of $\omega$ with $\beta$ at $f(=0.1,1$ \& 1.9) for type-2 1-D FPhCs

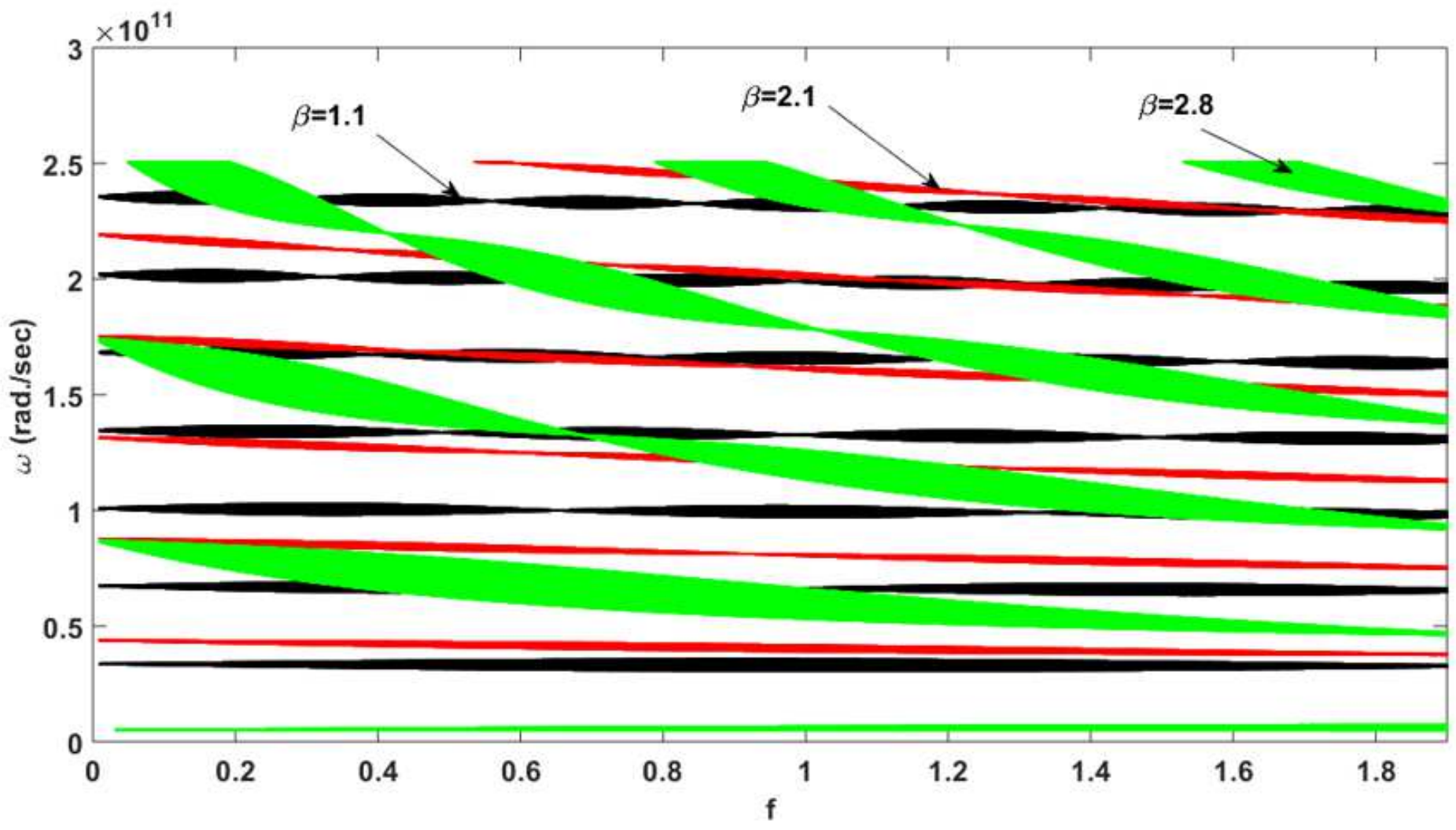

Figure 7 
Variation of $\nabla$ with $f$ at $\beta=1.1,2.1 \& 2.8$ for type-1 1-D FPhCs

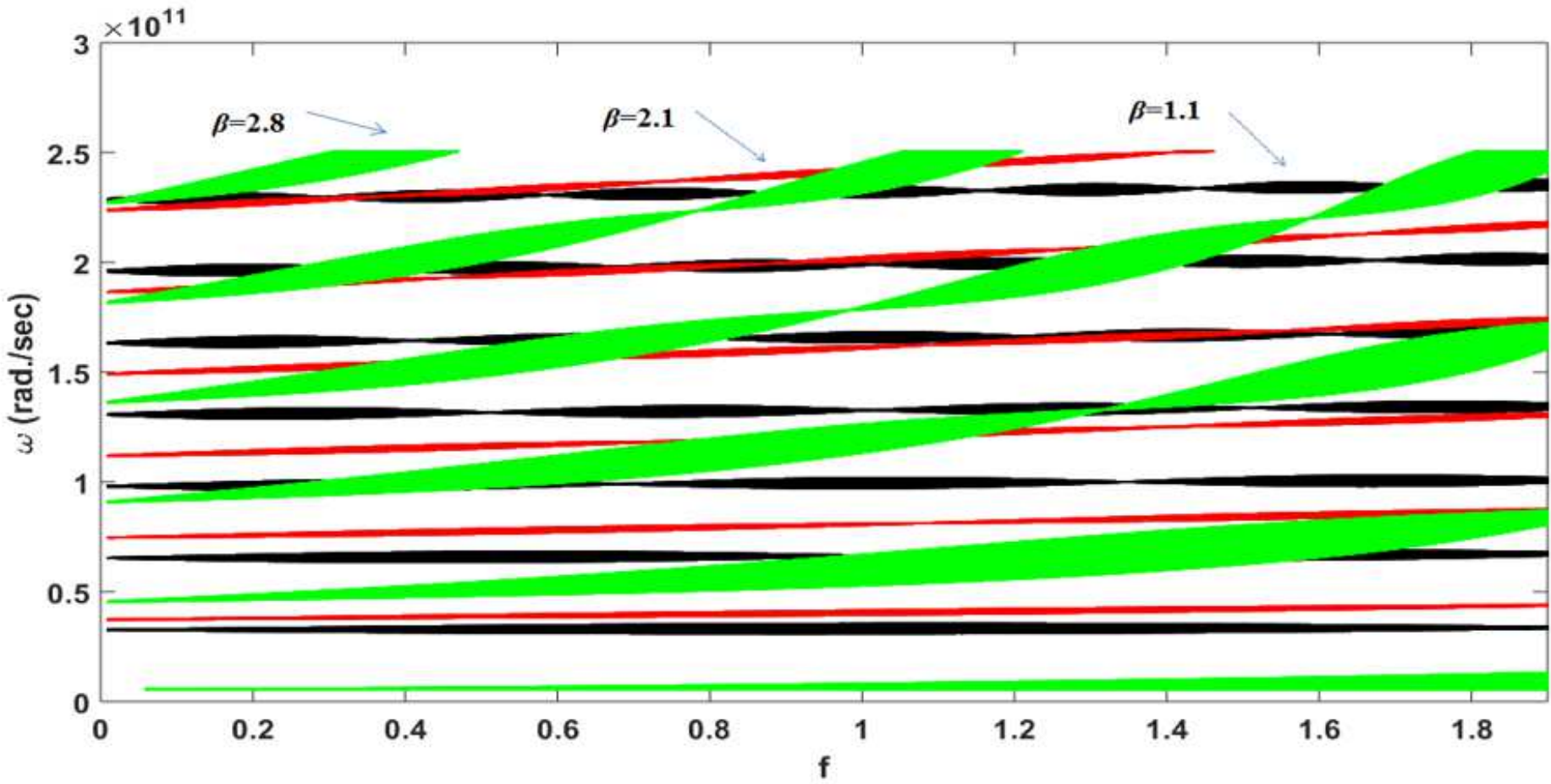

Figure 8

Variation of $\nabla$ with $f$ at $\beta=1.1,2.1 \& 2.8$ for type-2 1-D FPhCs

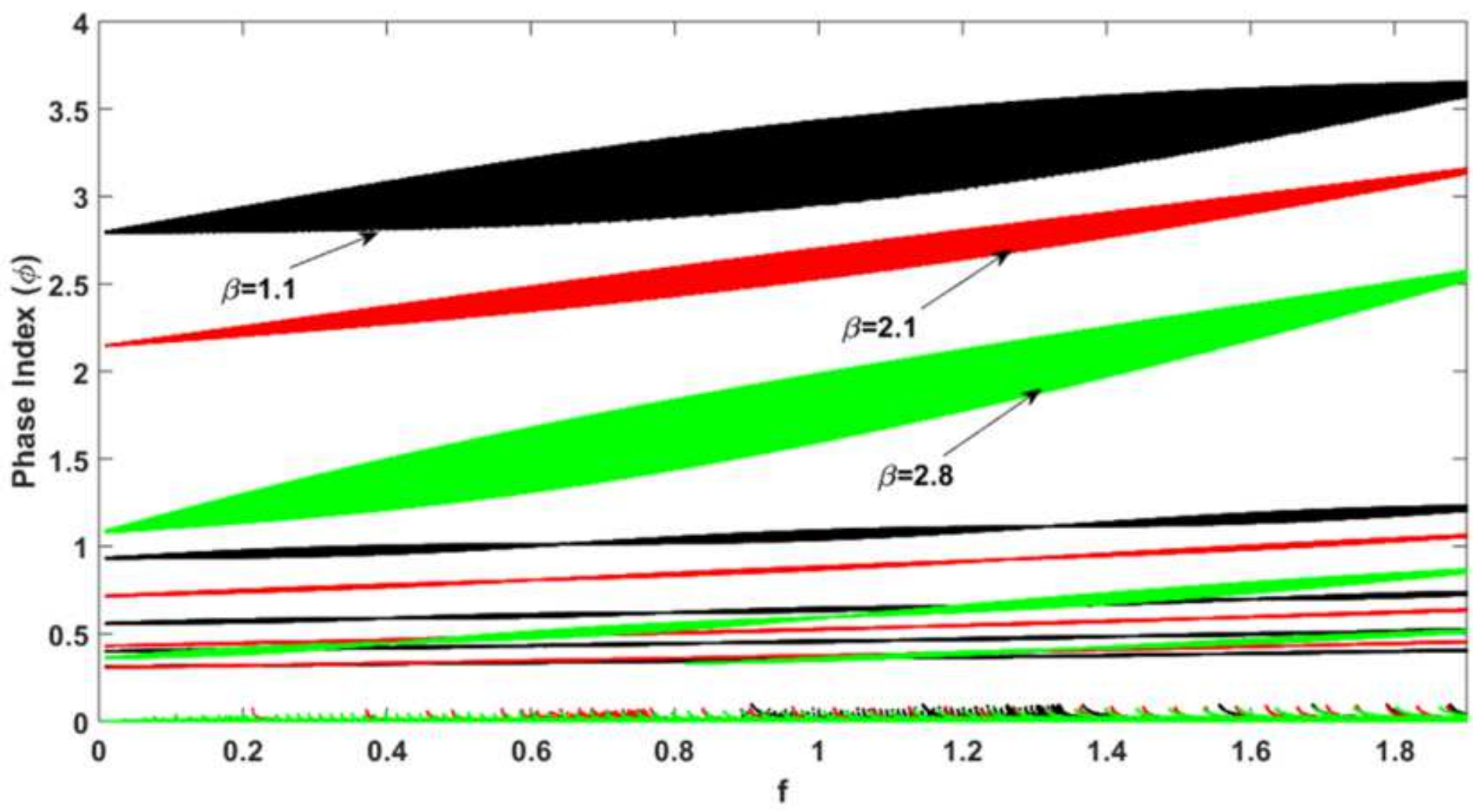

Figure 9 
Variation of $\nabla$ with $f$ at $\beta=(1.1,2.1 \& 2.8)$ for type-1 1-D FPhCs

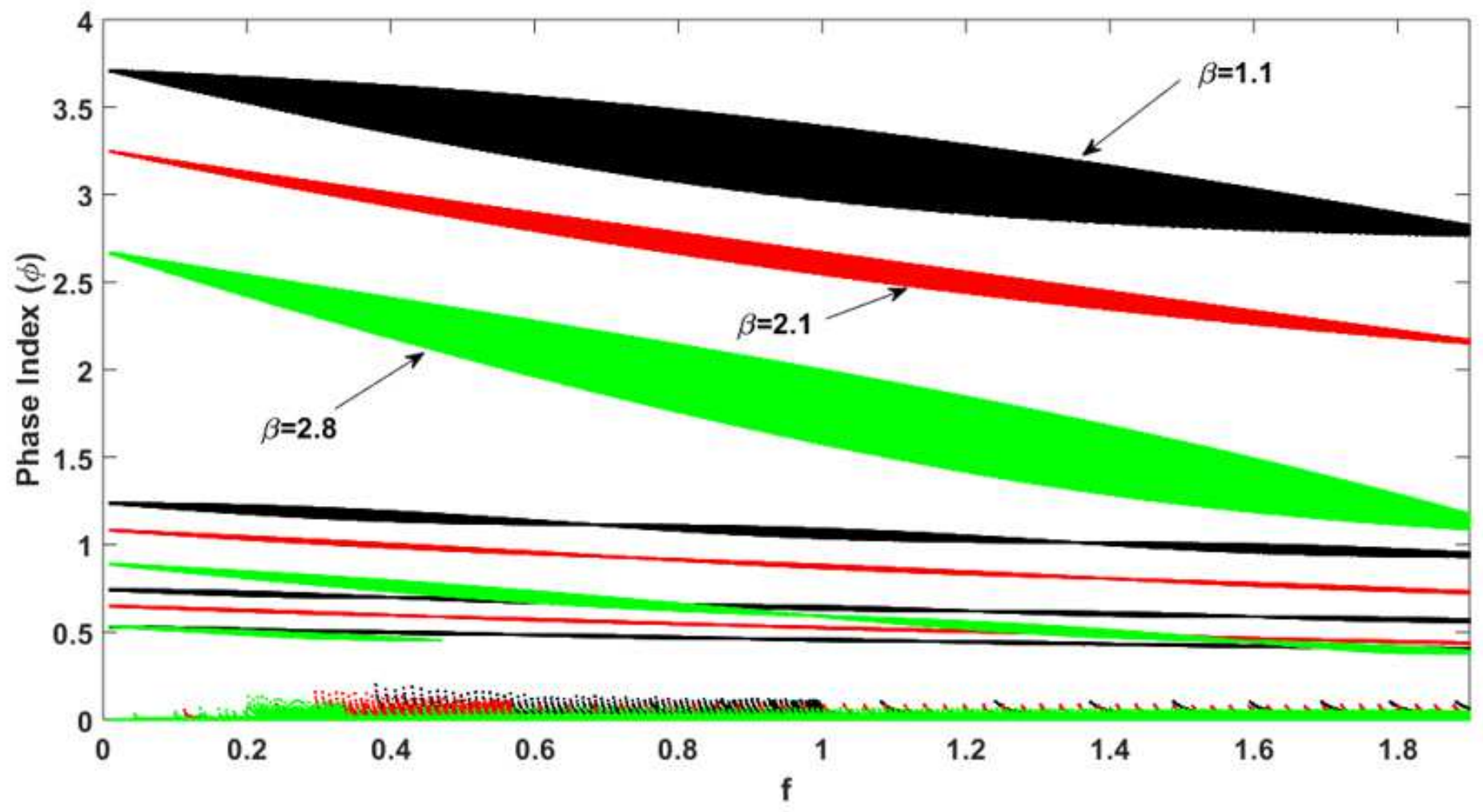

Figure 10

Variation of $\nabla$ with $f$ at $\beta=1.1,2.1 \& 2.8$ for type-2 1-D FPhCs

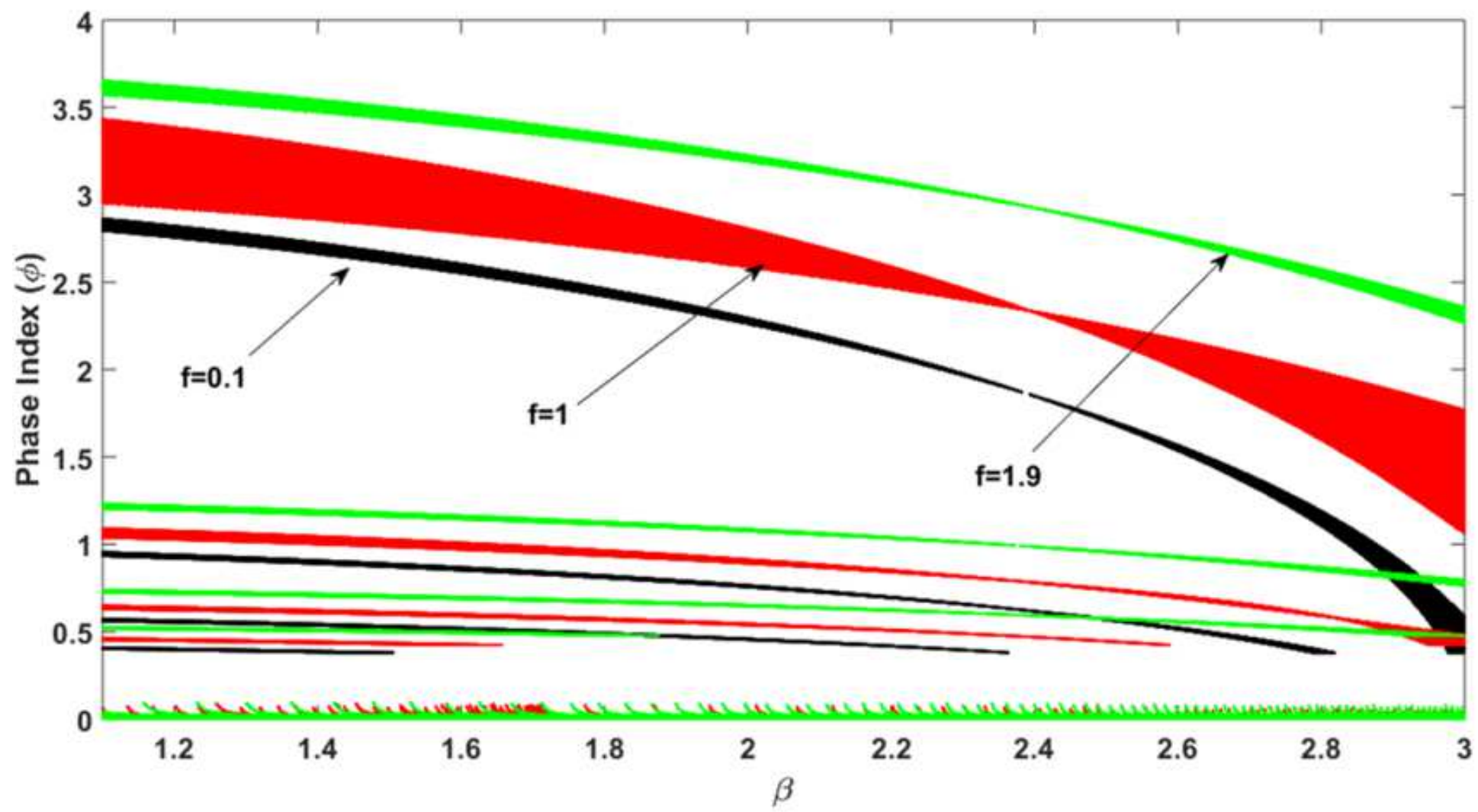




\section{Figure 11}

Variation of $\otimes$ with $\beta$ at $f=(0.1,1 \& 1.9)$ for type-1 1-D FPhCs

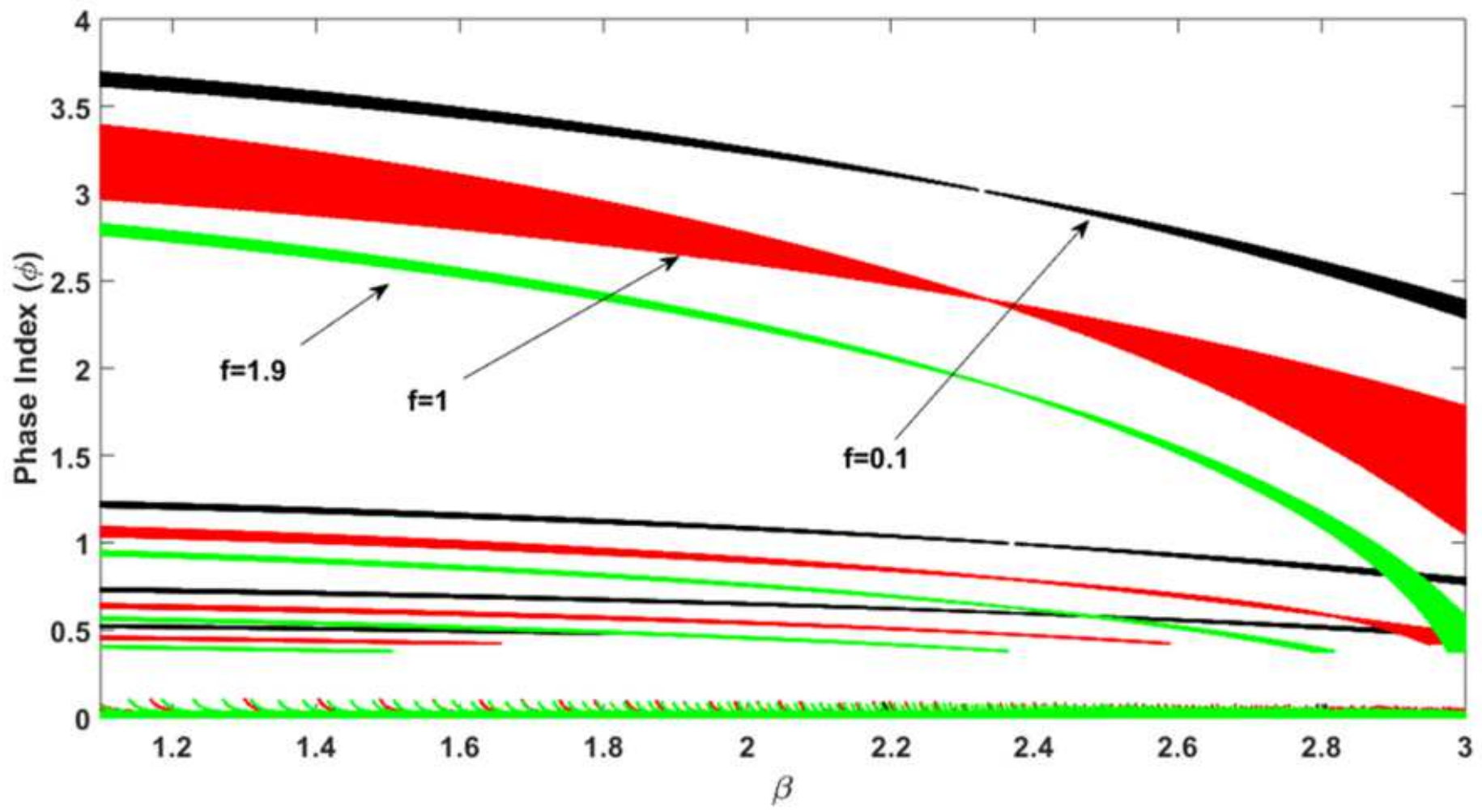

Figure 12

Variation of $\otimes$ with $\beta$ at $f=(0.1,1 \& 1.9)$ for type-2 1-D FPhCs

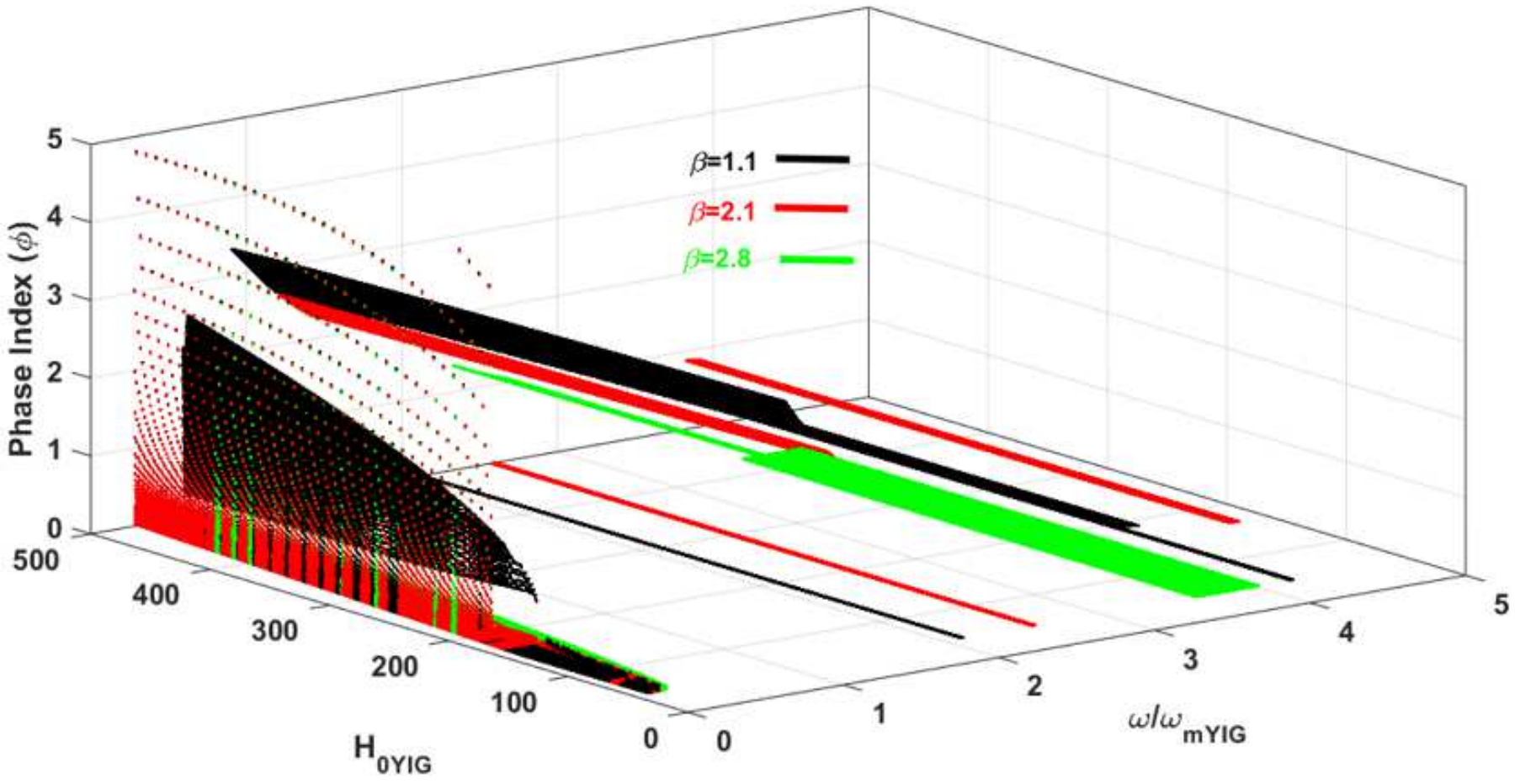


Figure 13

Variation of $\otimes$ with $\otimes H \otimes \_O Y I G$ at different $\beta$ with the normalized frequency $\left(\omega / \omega \_m Y I G\right)$ for type-1 1-D FPhCs

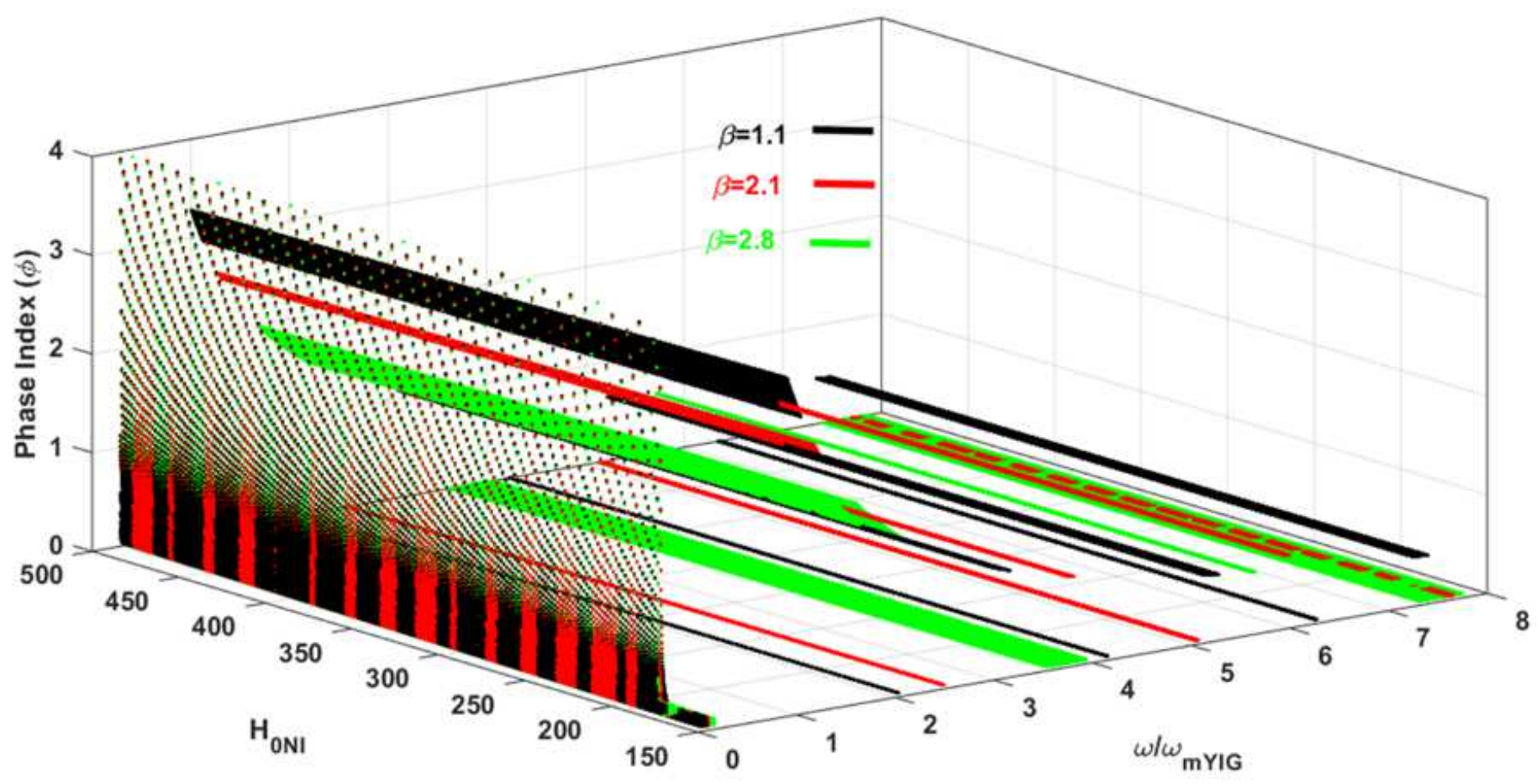

Figure 14

Variation of $\nabla$ with HOYIG at different $\beta$ with the normalized frequency $\left(\omega / \omega \_m Y I G\right)$ for type-2 1-D FPhCs 